\title{
School Supports for Reintegration Following a Suicide-Related Crisis: A Mixed Methods Study Informing Hospital Recommendations for Schools During Discharge
}

\author{
Marisa E. Marraccini ${ }^{1}$ (D) . Cari Pittleman ${ }^{1} \cdot$ Emily N. Toole $^{1} \cdot$ Megan R. Griffard $^{1}$
}

Accepted: 7 August 2021 / Published online: 2 October 2021

(C) The Author(s), under exclusive licence to Springer Science+Business Media, LLC, part of Springer Nature 2021

\begin{abstract}
The immediate period following psychiatric hospitalization is marked by increased risk for suicide behavior and rehospitalization. Because adolescents commonly return to school settings following hospital discharge, school-related stressors and supports are important considerations for psychiatric treatment and discharge planning. The current study aimed to inform recommendations provided by hospitals to schools to improve school reintegration practices by employing a concurrent, mixed-methods design. Specifically, we: (1) surveyed school professionals $(n=133)$ in schools varying in resource availability and populations in one southeastern state of the United States about supports and services provided to returning students; and (2) conducted in-depth interviews with a subset of these professionals $(n=19)$ regarding their perceptions of the hospital to school transition for youth recovering from suicide-related crises. Findings from survey responses indicated that, compared to schools located in urban and suburban areas, schools in rural areas were less likely to have school reintegration protocols for returning students. More generally, however, available interventions and modifications were relatively consistent across rural and urban/ suburban schools, schools serving high and low poverty communities, and schools with predominantly white and predominantly ethnic and racial minoritized student bodies. Key themes across interviews signify the importance of communication between stakeholders, the type of information used to develop re-entry plans, available school-based services for returning youth, and the need to mitigate stigma associated with mental health crises. Findings inform recommendations that can be provided by hospitals to schools to support adolescent recovery as they return to school following psychiatric hospitalization.
\end{abstract}

Keywords School reintegration - Suicide $\cdot$ Adolescents $\cdot$ Mental health policy . Psychoeducation

I think a lot of kids come back unequipped, and it's just the exact same. That's what sends them into the cycle again is that they don't have anything. They don't have any replace[ment] behaviors, and so they can just fall back into the same patterns. Then they don't wanna go back to the hospital because it was scary, and so

Marisa E. Marraccini

mmarracc@unc.edu

1 School of Education, University of North Carolina at Chapel Hill, Chapel Hill, USA 
they just try to stay under-more under our radar instead of dealing.... There's still a lot of follow-through, but I also-I gather that it's not enough.

\section{- School Professional}

Because schools are a primary discharge environment for adolescents hospitalized for a suicide-related crisis, an increasing number of studies have begun to explore best practice for supporting adolescents during school reintegration [1]. These foundational studies have surveyed school psychologists about existing practice [2], identified provider perceptions of facilitators and barriers to re-entry [3,4], and begun to centralize adolescent [5-7] and family [8] experiences during their return. Although findings from these studies have helped to inform school-related procedures for reintegration, they have rarely addressed hospital procedures for integrating school re-entry planning into their treatment and discharge process. And, as evidenced by the quote above, the current state of supports for adolescents recovering from a suicide-related crisis is "not enough."

A recent survey suggests that more than half of schools (62\%) may have a formal or informal reintegration procedure focused on supporting youth returning to school following a psychiatric hospitalization, with comparable rates of reintegration procedures across varying types of communities (e.g., rural and urban communities, high and low free and reduced lunch rates, and diverse ethnic and racial student body characteristics [2]). Protocols typically involve meetings with families, communication with hospital providers, and the development of a re-entry plan [2]. School re-entry plans are recommended to manage school-related stressors, identify supports and interventions, address the psychosocial climate of the school environment, establish a safety plan, and identify key individuals supporting the student $[2,3,5,6,9,10]$. Although school counselors have been identified as the most common professional involved in the process, a wide range of school professionals (e.g., administrators, other school support staff) may support returning youth $[2,9]$.

Collectively, practitioners and researchers have called for improved communication between hospitals, families, and schools during and following discharge [1, 9, 11]. Findings from research conducted with children and adolescents during psychiatric hospitalization suggest the need for inpatient procedures to address school-related issues, and also identify the importance of discharge planning that attends to the transition from hospital to school [6]. Such supports and services should be tailored to the individual needs and circumstances of the patient [1], as well as the individual context of the school. Although school and hospital providers have noted the importance of providing schools with discharge summaries and recommendations for supporting student reintegration, school professionals have described instances where recommendations provided by hospitals may be unrealistic in school settings [12]. For example, certain services (e.g., therapeutic schools and specialized instruction) may only be allowable if a student has an Individualized Education Plan (IEP) for special education, and resource limitations in schools with higher levels of poverty or schools located in rural areas may inhibit the availability of specific supports and services (e.g., school-based mental health services). Therefore, identifying supports and services that are available and feasible to implement in school settings could increase the utility of hospital recommendations to schools.

Common services available to support students upon their return may include individual counseling, on-site tutoring, and support with time management [2]. Commonly 
available accommodations may include a universal pass to see a counselor, flexibility in time of arrival and departure from school, and extended academic completion deadlines [2]. Although the extent to which the availability of these supports and services may vary across communities remains unclear, research identifying variability in school characteristics more broadly (e.g., rurality, poverty, student demographic characteristics) has painted a relatively clear picture of differences across communities. Put simply, compared to other schools, rural and high-poverty schools have fewer resources to serve students (e.g., [13-15]) and myriad obstructions prevent ethnic and racial minoritized students from accessing mental health care $[16,17]$.

Educators staffing high-poverty schools are more likely to have lower qualifications than their counterparts in low-poverty schools [13]. Student mental health and counseling resources, as well as the perceptions of their utility and efficacy, may also vary across school contexts [18]. Fortunately, schools with large populations of economically disadvantaged and minoritized students that comply with the American School Counselor Association (ASCA) recommended student-counselor ratio (250:1) have higher graduation and attendance rates and lower levels of disciplinary incidents [19,20]. Yet, as Monteiro-Leitner and colleagues [21] found, rural school professionals are more likely to spend time on non-counseling duties, such as supervisory duties, clerical duties, special education programs and services, and administrative duties; and school districts rarely meet the prescribed ASCA standards [22].

The rate of adolescent death by suicide is double in rural areas compared to urban ones, especially among males [23], and the approaches rural and urban schools take for suicide prevention programs may differ. Schorr et al. [24] recommended rural schools implement programs that target the specific factors related to suicide that may be more prevalent in rural regions. These factors include: a lack of access to mental and physical health resources in the community, rural ideologies regarding mental health and distress, lack of community support for specific demographic subgroups (e.g., LGBTQIA + students), geographic and personal isolation, high rates of substance abuse, and exposure to violence or sexual violence [24, 25]. In urban contexts, training teachers and mental health professionals to respond to crises is associated with reductions in suicidal behavior [26, 27].

Ample research has also demonstrated lower utilization of mental health services for ethnic and racial minoritized youth (e.g., [28]), including among adolescents experiencing suicidal thoughts and behaviors $[29,30]$. Obstructions to service include stigma and shame, reliance on non-medical mental health supports (e.g., religious communities, family networks), affordability and accessibility of services, a dearth of culturally relevant clinicians and interventions, and systemic discrimination [16, 17]. Schoolbased mental health services are thought to address these impediments given that some research has demonstrated equitable service provision across ethnic/racial groups [31-33] and similar outcomes for suicide prevention initiatives regardless of student ethnicity/race (e.g., [34-36]). Yet, even when schools can mitigate ethnic/racial disparities, obstructions related to community mental health remain. For example, a study by Guo et al. [37] found that a school-based depression screener identified a proportional number of non-white students, but follow-through and parent consent were lower for Latinx and Asian students. School mental health professionals have reported that their provision of services to ethnic and racial minoritized youth can be impeded by cultural barriers, lack of interpretation services, and limited training for diverse populations of students [38]. 


\section{Aims of the Current Study}

Irrespective of differences in settings and communities, school-based services remain critical for supporting youth who may not otherwise have access to behavioral and mental health services. Schools are often the de facto mechanism for treatment of youth with psychiatric concerns [39]. Yet, hospital recommendations for school reintegration following a suiciderelated crisis need to be tailored according to the individual needs of patients and the context of their school. To date, no studies have systematically investigated school-related supports and services provided to students as they return to school following psychiatric hospitalization based on resource availability or population served, or applied a mixed-methods approach to understand stakeholder perspectives about available supports and services. Therefore, the present study employed a concurrent, triangulation design to inform improved hospital discharge planning and recommendations provided to schools. Specifically, this study aimed to (1) identify school-based supports and services available to adolescents returning from psychiatric hospitalization that are (a) generalizable across most school districts and (b) unique to particular school districts based on resource availability and population served; and (2) triangulate findings to inform recommendations for schools provided by hospitals to improve the school re-entry process.

\section{Methods}

This study was part of a larger study that includes survey data collected from school professionals $(n=133)$ and qualitative interviews conducted with a range of key stakeholders that included adolescents $(n=19)$, parents $(n=19)$, school professionals $(n=19)$, and hospital professionals $(n=7)$. The aim of the larger study is to develop school re-entry guidelines for adolescents hospitalized for a suicide-related crisis based on multiple stakeholder perceptions [7]. For the current paper, we focus on school professional surveys and interviews that identify school-related supports, services, and experiences for adolescents returning to school following hospitalization for a suicide-related crisis.

Using a concurrent, triangulation design, quantitative (phase 1) and qualitative (phase 2) data were collected simultaneously, and data were merged during interpretation and analysis [40]. Quantitative data, addressing supports and services delivered to students returning from hospitalization, were collected from school professionals across one southeastern state of the United States (US; $n=133$ ). Qualitative data, collected from interviews conducted with a purposive subsample of survey participants $(n=19)$, were interpreted alongside qualitative data to inform hospital recommendations for schools.

\section{Participants}

A total of 35 school districts and one charter school approved dissemination of recruitment information to student support professionals in their school districts. School professionals were invited by way of email to complete a confidential survey focused on school reintegration procedures and protocols. If allowable by school district, school professional survey respondents were compensated $\$ 5$ for completing the survey. During the consent process for quantitative surveys, participants indicated if they were interested in completing 
a qualitative interview about their perceptions of and experiences with school re-entry. School professional interview participants were compensated \$20 for their participation in the interview (if allowable by participating districts).

Eligibility criteria for participation included working as a high school professional (e.g., school counselor, school psychologist) in one southeastern state of the US and experience with supporting adolescents with suicide-related risk. A total of 172 individuals from participating school districts completed consent procedures and opened the survey, of whom 133 answered the study's primary question addressing school reintegration protocols for students hospitalized for suicide-related thoughts and behaviors. Participants were from 27 different school districts, including 84 different schools (30 schools were reported on by more than one participant). Participants included school psychologists (14.3\%), school social workers (12.0\%), school counselors (29.3\%), school administrators (7.5\%), school nurses (12.0\%), regular education teachers (13.5\%), special education teachers (9.0\%), and other (5.3\%). Participants $(n=97)$ reported working between 0 and 21 years (mean $=7.2$ years, standard deviation $[\mathrm{SD}]=5.8$ years) at their current school and 1 and 32 years in the profession (mean $=12.8$ years, $\mathrm{SD}=7.3$ ). A total of 98 respondents self-identified their demographic information (note that for a total of 10 participants, this information was not collected due to district restrictions). Of 97 reporting their demographics, $88.7 \%$ identified as female and $97.9 \%$ reported that their ethnicity was non-Latinx or non-Hispanic (with one additional participant preferring not to answer). Regarding self-reported race $(n=98)$, participants identified as white $(89.8 \%)$, Black or African American (6.1\%), American Indian or Alaskan Native (2.0\%), or other (2.0\%).

A purposive subsample of survey respondents, selected to represent a range of professions working in districts across the state, participated in in-depth interviews $(n=19)$. Participants were from a total of 9 school districts and at least 18 different high schools (with some working in multiple schools). Participant interviewees were school psychologists $(n=4)$, school counselors $(n=4)$, social workers $(n=4)$, nurses $(n=2)$, teachers $(n=2)$, a principal $(n=1)$, a special education teacher $(n=1)$, and other $(n=1)$. Fifteen participants identified as female and four as male. One participant identified as Hispanic or Latinx and indicated "other" for race, two participants identified as Black or African American, and the remaining 15 identified as white.

\section{Measures}

\section{Surveys}

Participants completed a battery of self-report questionnaires that included participant and school demographic characteristics, a modified version of the School Reintegration Questionnaire [2], and the Authoritative School Climate Survey (ASCS; [41]). For the current study, only demographic questions and the modified School Reintegration Questionnaire were analyzed.

The School Reintegration Questionnaire, which was developed to explore perceptions about school re-entry procedures following psychiatric hospitalization, was administered to participating school professionals. This survey was developed iteratively based on stakeholder feedback and then pilot tested with school psychologists in a study examining existing processes and protocols for supporting school re-entry following psychiatric hospitalization [2]. The original survey includes 46 questions addressing school and community mental health services (11 items), school reintegration protocols (19 items), and quality of school reintegration protocols (4 items). 
After refining the survey based on feedback from a survey methodologist and findings from the previous study, we only included 9 questions related to school reintegration protocols and 19 questions addressing school and community mental health services (each with multiple sub-sections; the administered survey is available in Supplementary Materials). The questionnaire also includes demographic questions (school level and grades, rurality, gender, race, ethnicity, free/reduced lunch status of student population, number of students in school, school type, proximity to and quality of mental health services). Most of the items in the survey are descriptive in nature (i.e., precluding psychometric property analysis).

\section{Interviews}

A semi-structured qualitative interview guide addressed four broad areas related to adolescent hospitalization and school re-entry: (a) school experiences prior to hospitalization; (b) school experiences and considerations during hospitalization; (c) school re-entry experiences and processes; and (d) information sharing between hospitals and schools. For the current study, we focused on themes related to descriptions of school re-entry processes, part of (c) school re-entry experiences and processes. Following interviews, debrief summaries were completed to summarize key themes. Interviews were conducted in-person, virtually, or by phone, according to participant preference, ranging from 35 to 100 minutes long.

\section{Data Analyses}

\section{Quantitative Analyses}

We conducted descriptive analyses to identify commonly endorsed procedures for re-entry, components of re-entry considerations, individuals involved in re-entry, services available to students returning to school, and accommodations available to students returning to school. Available services and accommodations were then analyzed in a matrix according to school district county poverty level (less than $20 \%$ vs. $20 \%$ or more at or above the poverty level) and urbanicity (suburban or urban compared to rural), as well as individual school ethnic and racial student body demographics $(50 \%$ or more ethnic or racial minoritized students compared to $50 \%$ or more white). Chi-square analyses were conducted to compare differences in availability of protocols and procedures by poverty and urbanicity. Analyses were conducted using SPSS [42].

Poverty level and urbanicity were determined based the county where each school district is located. County-level poverty data was obtained from the Economic Research Service, U.S. Department of Agriculture [43] indicating the percent of children ages 0-17 in poverty. School districts were categorized into binary poverty levels: (a) districts providing services in counties in which $20 \%$ or more of families are at or above the poverty level $(n=44 ; 34.1 \%)$ were considered high and (b) districts providing services in counties in which less than $20 \%$ of families are at or above the poverty level $(n=85 ; 65.9 \%)$ were considered low. Urbanicity was determined based on county-level density calculations from a local database. Rural districts $(n=86,64.7 \%)$ were compared to urban or suburban districts $(n=47,35.3 \%)$. Student body ethnic and racial characteristics were determined based on publicly available data provided by the state's department of education for each school; when unavailable by the state (e.g., in the case of charter schools), self-report data provided by participants were used. School demographics were coded into binary categories 
of those including predominantly ethnic and racial minoritized student bodies (i.e., $50 \%$ or more; $n=45,35.4 \%$ ) compared to those with predominantly non-Latinx white Eurocentric student bodies $(n=82,64.6 \%)$.

\section{Qualitative Analyses}

One open-ended question on the School Reintegration Survey part of phase 1 instructed participants to describe their school re-entry protocol. Responses were coded using content analysis, a systematic method for segmenting text into content categories [44]. The first author read through each response and created a coding structure based on emergent themes. Following a training, the first and third author coded each response using the coding structure. All items were double coded and in the case of discrepancies, the authors met to reach consensus, refining the coding structure as needed.

Qualitative interviews were audio recorded and transcribed by a professional transcription company. Cleaned transcriptions of interviews were redacted of identifying information and then entered into NVivo 12 Pro qualitative data analysis software program [45]. We conducted applied thematic analysis, which is a systematic and rigorous approach to identifying themes in text that is based on multiple theoretical and methodological perspectives (e.g., inductive thematic analysis, grounded theory, and phenomenology [46]), to analyze the transcripts. We first developed a coding structure for interviews based on the interview agenda and made iterative changes to it based on emerging themes. Two trained researchers independently read transcripts and identified themes, meeting regularly to come to consensus about the coding structure. All interviews were double coded, resulting in summaries and illustrative quotes to show common themes for final codes.

\section{Phase 1 Results}

Survey results are presented in the following order: (1) frequency of hospitalization, (2) prevalence of school reintegration protocols, (3) descriptions of protocols based on openended text responses, (4) procedures and components within protocols, (5) key individuals supporting reintegration, and (6) services and accommodations to support reintegration. Note that because qualitative themes drawn from descriptions of protocols (3) were analyzed alongside descriptive results, a subset of themes are presented alongside descriptive information presented in subsequent sections.

\section{Frequency of Hospitalization}

Participants $(n=119)$ estimated the number of students they referred for psychiatric care during their most recent school year. On average, 9.2 students were referred per year $(\mathrm{SD}=11.6$; range from 0 to 70$)$, with most participants $(71.4 \%)$ reporting a range between 1 and 10.

\section{Prevalence of School Reintegration Procedure or Protocol}

A total of 133 participants answered the question asking if their school had a protocol or procedure for school reintegration. A total of $41.4 \%$ reported having no procedure, 
with the remaining 58.6\% reporting having a formal (written) protocol $(32.3 \%$ ) or an informal (not written) protocol (25.3\%). Suburban and urban districts (70.2\%) were significantly more likely to report having a re-entry plan compared to rural districts $(52.3 \%$; $\left.\chi^{2}=4.01, p=.045\right)$. Results were not significantly different for districts with high compared to lower rates of poverty $\left(\chi^{2}=2.83, p=.09\right)$, or for schools comprising majority ethnic and racial minoritized students compared to those comprising majority white students $\left(\chi^{2}=1.82, p=.18\right)$. Of the 75 participants who answered the question about the level at which their re-entry protocol was established, $37.3 \%$ reported that this procedure was set by their school and $62.7 \%$ reported it was set by the district. We also assessed level of agreement between participants reporting on procedures within the same school, with responses indicating disagreement between having a procedure (formal or informal) and not having a procedure in 15 of the 30 schools (50\%).

\section{Open-Ended Description of School Reintegration Procedure or Protocol}

Participants reporting that their school had a formal or informal re-entry protocol were next asked to provide a brief description of their school's protocol by typing into an open text box. A total of 71 participants described their school's procedures. Responses were coded inductively, revealing six main categories: (1) identification of a specific protocol for suiciderelated risk or school reintegration; (2) description or identification of re-entry meetings; (3) individuals involved in supporting re-entry; (4) information informing how best to support returning students (e.g., precipitants, medication); and (5) plans or actions taken to support returning students (e.g., changing schedules, checking in).

Regarding the first category (protocols), a handful of participants $(n=5)$ indicated that they were not sure or unaware of the specific procedures in their school; however, 12 identified having a specific protocol for re-entry (e.g., "We have a formalized reintegration protocol for students who have had extended hospital stays"). A number of participants $(n=9)$ described their risk assessment protocol (e.g., "We evaluate the students and determine if there is suicidal ideation and then determine their level between low medium and high") instead of a re-entry protocol, signifying some confusion around differences between risk assessments/referrals and reintegration. The remainder of categories reflect overlapping results from the close-ended questions on the surveys and are presented in the subsequent sections according to content (and can also be found in Supplementary Table S1).

\section{Specific Procedures and Components of School Reintegration Protocols}

Participants who reported having a formal or informal school re-entry protocol next identified specific procedures that were part of their school's protocol, as well as specific components considered within the school re-entry planning process (see Tables 1 and 2, respectively). For each selection, participants indicated if the option was mandatory, optional, or unavailable. Majority of respondents (more than 60\%) endorsed meetings with the family and student and the development of a re-entry plan as mandatory procedures for re-entry. Indeed, 36 participants also described having a re-entry meeting and 13 described documenting a re-entry plan in their open-ended responses. Although phone calls between the hospital and school were commonly endorsed as optional procedures $(63.6 \%$ indicated it as optional), in-person visits were commonly reported as not available (43.8\% and $46.9 \%$ indicated that in-person visits by school staff to hospital and vice versa were not available). 
Table 1 Procedures within school reintegration protocols

\begin{tabular}{lllll}
\hline Procedures for Re-Entry & $\mathrm{N}$ & Mandatory & Optional & Not Available \\
\hline Phone communication with hospital staff & 66 & $9(13.6)$ & $42(63.6)$ & $15(22.7)$ \\
In person visits by school staff to the hospital & 64 & $1(1.6)$ & $35(54.7)$ & $28(43.8)$ \\
In person visits by hospital staff to the school & 64 & $0(0.0)$ & $34(53.1)$ & $30(46.9)$ \\
Development of individualized re-entry plan & 66 & $39(59.1)$ & $23(34.8)$ & $4(6.1)$ \\
Referral for Special Education/504 Evaluation & 64 & $6(9.4)$ & $54(84.4)$ & $4(6.3)$ \\
Meeting with family about re-entry needs & 69 & $44(63.8)$ & $23(33.3)$ & $2(2.9)$ \\
Meeting with student about re-entry needs & 69 & $45(65.2)$ & $22(31.9)$ & $2(2.9)$ \\
\hline
\end{tabular}

Numbers in this table represent frequencies $(n)$, with percents $(\%)$ in parentheses

Commonly identified considerations within school re-entry plans that were mandatory included hospital evaluations or recommendations (53.0\%), previous school-based evaluations $(51.6 \%)$, recommendations by parents or other family members $(51.5 \%)$, and recommendations by students (45.5\%; see Table 2). Recommendations from teachers, staff (school counselors, school social workers, school psychologists) and outside mental health professionals were commonly endorsed as optional considerations. Fourteen responses from the open-ended text also suggested that school professionals sought out or integrated hospital discharge summaries and recommendations into their decisionmaking for the re-entry plan, with one describing it as mandatory.

Although a separate school for transitioning from the hospital was relatively uncommon within school re-entry plans $(61.5 \%$ reported it was not available and only two responses from open-ended responses indicated the availability of an alternative school prior to reentry), more than half of those with a re-entry protocol (56.2\%) reported a gradual return

Table 2 Components considered within school re-entry planning

\begin{tabular}{lllll}
\hline School Re-Entry Plan Components & $\mathrm{N}$ & Mandatory & Optional & Not Available \\
\hline Consideration of hospital evaluations/recommendations & 66 & $35(53.0)$ & $26(39.4)$ & $5(7.6)$ \\
Consideration of previous school based evaluations & 65 & $33(50.8)$ & $27(41.5)$ & $5(7.7)$ \\
Recommendations provided by Teachers & 65 & $20(30.8)$ & $41(63.1)$ & $4(6.2)$ \\
Recommendations provided by School Counselors & 65 & $32(49.2)$ & $31(47.7)$ & $2(3.1)$ \\
Recommendations provided by School Social Workers & 66 & $25(37.9)$ & $35(53.0)$ & $6(9.1)$ \\
Recommendations provided by School Psychologists & 65 & $15(23.1)$ & $42(64.6)$ & $8(12.3)$ \\
Recommendations provided by outside mental health professionals & 66 & $24(36.4)$ & $39(59.1)$ & $3(4.5)$ \\
Recommendations provided by parents or other family members & 66 & $34(51.5)$ & $29(43.9)$ & $3(4.5)$ \\
Recommendations provided by student & 66 & $30(45.5)$ & $33(50.0)$ & $3(4.5)$ \\
Recommendations provided by others & 65 & $8(12.3)$ & $53(81.5)$ & $4(6.2)$ \\
Separate school for transition prior to return to classes & 65 & $3(4.6)$ & $22(33.8)$ & $40(61.5)$ \\
Gradual return to school using transition space outside of school & 64 & $3(4.7)$ & $33(51.6)$ & $28(43.8)$ \\
$\quad$ (e.g., separate facility for academic and social-emotional & & & & \\
$\quad \begin{array}{llll}\text { support) } \\
\text { Gradual return to academic classes using transition space within }\end{array}$ & 65 & $2(3.1)$ & $46(70.8)$ & $17(26.2)$ \\
$\quad \begin{array}{l}\text { school (e.g., separate area for academic and social-emotional } \\
\text { support) }\end{array}$ & & & &
\end{tabular}

Numbers in this table represent frequencies $(n)$, with percents $(\%)$ in parentheses 
Table 3 Key individuals involved in school reintegration

\begin{tabular}{llllll}
\hline Individuals Involved in Re-Entry & $\mathrm{N}$ & Never & Sometimes & Always & N/A \\
\hline School Psychologist & 107 & $38(35.5)$ & $43(40.2)$ & $7(6.5)$ & $19(17.8)$ \\
School Counselor & 109 & $0(0.0)$ & $33(30.3)$ & $71(65.1)$ & $5(4.6)$ \\
Principal & 108 & $8(7.4)$ & $52(48.1)$ & $43(39.8)$ & $5(4.6)$ \\
Vice Principal or Assistant Principal & 106 & $9(8.5)$ & $63(59.4)$ & $24(22.6)$ & $10(9.4)$ \\
School Nurse & 108 & $14(13.0)$ & $72(66.7)$ & $16(14.8)$ & $6(5.6)$ \\
School Social Worker & 109 & $6(5.5)$ & $63(57.8)$ & $24(22.0)$ & $16(14.7)$ \\
Special Education Teacher(s) & 108 & $14(13.0)$ & $79(73.1)$ & $4(3.7)$ & $11(10.2)$ \\
Regular Education Teacher & 108 & $19(17.6)$ & $68(63.0)$ & $13(12.0)$ & $8(7.4)$ \\
Parents or Family & 108 & $1(0.9)$ & $35(32.4)$ & $68(63.0)$ & $4(3.7)$ \\
Student & 107 & $2(1.9)$ & $36(33.6)$ & $65(60.7)$ & $4(3.7)$ \\
\hline
\end{tabular}

Numbers in this table represent frequencies $(n)$, with percents $(\%)$ in parentheses

to school using a transition space outside of school (e.g., separate facility for support) as optional or mandatory, and nearly three-quarters $(73.9 \%)$ reported a gradual return to school using a transition space within school as optional or mandatory. Twelve participants indicated that their school considered gradual returns using other services, identifying online learning programs and homebound services $(n=8)$, partial outpatient programs $(n=2)$, tailoring services to the individual $(n=1)$, and no services $(n=1)$.

A number of participants $(n=19)$ also identified the type of information considered within re-entry planning when describing their school re-entry procedure in the openended text response (see Supplementary Table S1). Multiple $(n=14)$ described wanting broad information, such as how best to support the student. A handful described the importance of understanding specific information needed to keep the student safe, such as the student's triggers and warning signs $(n=5)$, reasons for hospitalization $(n=3)$, information about follow-up care $(n=3)$, and medications $(n=3)$. Six responses indicated that school professionals considered coping strategies for the student to use during their school day (primarily recommended by the hospital). Additional considerations not addressed in the close-ended survey responses that emerged in the open-ended responses regarding re-entry planning included the development of a safety plan $(n=11)$ and the request for a release to speak with an outside clinician $(n=4)$.

\section{Key Individuals Supporting School Reintegration}

As shown in Table 3, respondents were also asked to identify how frequently specific individuals were involved in the re-entry process. In addition to students and families (identified as always being involved by $63.0 \%$ and $60.7 \%$ of respondents, respectively), the most commonly endorsed professionals involved were school counselors $(65.1 \%$ indicated they were always involved) and school principals (39.8\% indicated they were always involved). Open-ended responses coded to identify individuals involved in the process were similar, with families $(n=31)$, students $(n=34)$, counselors $(n=28)$, and administrators $(n=20)$ commonly identified. Participants were also asked to indicate how often a representative from the school communicates with the hospital to support re-entry. Out of the 107 participants responding to this question, the majority $(n=84,78.5 \%)$ indicated that a member of 
Table 4 Available services to returning students

\begin{tabular}{|c|c|c|c|c|c|}
\hline \multirow[b]{2}{*}{ Services } & \multirow[b]{2}{*}{$\mathrm{N}$} & \multirow[b]{2}{*}{ Available } & \multicolumn{3}{|c|}{ Requirements } \\
\hline & & & None & IEP & 504 \\
\hline Off-site tutoring & 102 & $17(16.7)$ & $14(82.4)$ & $3(17.6)$ & $2(11.8)$ \\
\hline On-site tutoring & 106 & $79(74.5)$ & $76(96.2)$ & $2(2.5)$ & $2(2.5)$ \\
\hline Peer mentoring programs & 103 & $31(30.1)$ & $30(96.8)$ & $1(3.2)$ & (0) \\
\hline Adult mentoring programs & 101 & $24(23.9)$ & $24(100)$ & (0) & (0) \\
\hline $\begin{array}{l}\text { Check in/Check out (e.g., regular check ins with students } \\
\text { by adult) }\end{array}$ & 105 & $95(90.5)$ & $91(95.8)$ & $2(2.1)$ & $1(1.1)$ \\
\hline Self-monitoring instruction & 101 & $62(61.4)$ & 57 (91.9) & $3(4.8)$ & $1(1.6)$ \\
\hline $\begin{array}{l}\text { Transition space within school (e.g., separate area for } \\
\text { academic and social-emotional support) }\end{array}$ & 104 & $74(71.2)$ & $61(82.4)$ & $9(12.2)$ & $10(13.5)$ \\
\hline $\begin{array}{l}\text { Transition space outside of school (e.g., separate facility } \\
\text { for academic and social-emotional support) }\end{array}$ & 104 & $25(24.0)$ & $18(72.0)$ & $6(24.0)$ & $6(24.0)$ \\
\hline Group counseling & 99 & $31(31.3)$ & $31(100)$ & (0) & (0) \\
\hline Individual counseling & 102 & $95(93.1)$ & $92(95.8)$ & $1(1.1)$ & $1(1.1)$ \\
\hline Social skills groups & 97 & $35(36.1)$ & $35(100)$ & (0) & (0) \\
\hline Support with time management/ assignment make-up & 101 & $90(89.1)$ & $80(88.9)$ & $7(7.8)$ & $6(6.7)$ \\
\hline Personalized Early Outreach & 101 & $59(58.4)$ & 57 (96.6) & $1(1.7)$ & $1(1.7)$ \\
\hline
\end{tabular}

Numbers in this table represent frequencies $(n)$, with percents $(\%)$ in parentheses. Participants could select multiple responses for requirements, so total percent may exceed $100 \%$. IEP $=$ Individualized Education Plan

the school sometimes communicated with the hospital (and only 7 [6.5\%] indicated never and 16 [15.0\%] indicated always).

\section{Services and Accommodations to Support Reintegration}

Table 4 displays the types of services and Table 5 displays the types of accommodations available to students following a psychiatric hospitalization. Participants were instructed to identify which, if any, were available, and also whether or not an Individualized Education Plan (IEP) or 504 Plan $^{1}$ were required to receive services and accommodations. The most commonly identified services available to all students included check-in/check-out $(86.7 \%)$, individualized counseling (90.2\%), and support with time management and assignment completion (79.2\%). Most services were available to all students, irrespective of their special education status.

Commonly reported accommodations, shown in Table 5, included receiving a universal pass to leave class as needed $(88.5 \%)$, reduced assignments or workloads $(90.4 \%)$, extended deadlines for assignments (98.1\%), missing work forgiveness (91.4\%), opportunities to take tests in quiet locations $(81.4 \%)$, and opportunities to retake tests

\footnotetext{
${ }^{1}$ Individualized Education Plans (IEP) are part of the Individuals with Disabilities Education Act (IDEA), and serve as a formal plan for a child's special education experience; IEPs allow for both accommodations (changes to the learning environment) and specialized instruction that are addressed by a special education teacher. 504 plans are part of Sect. 504 of the Rehabilitation Act, and serve as a formal plan to provide support and eliminate barriers for a student with a disability; 504 plans allow for supports and accommodations (but not specialized instruction).
} 


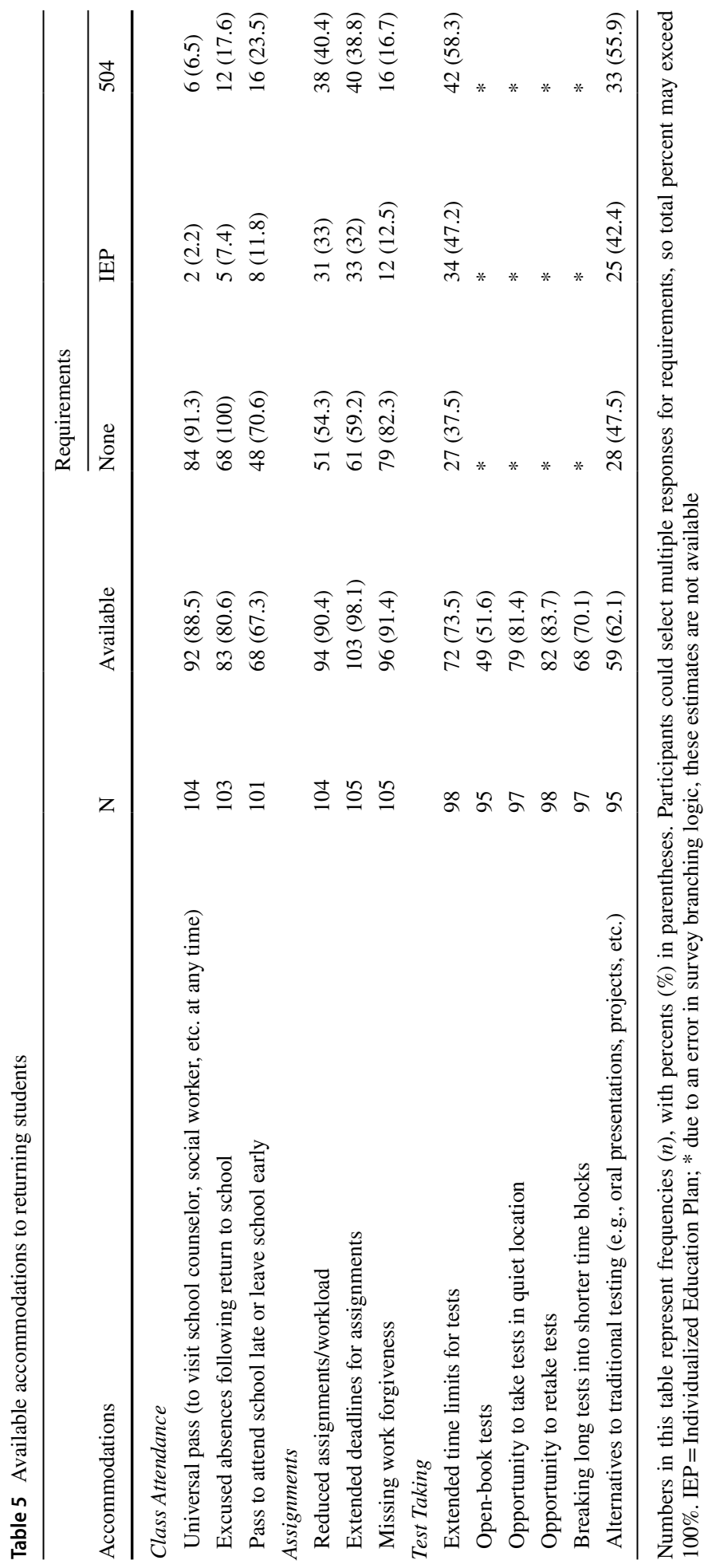


(83.7\%). Although offering a universal pass and missing work forgiveness appeared to be available to most students (irrespective of special education status), many of the other accommodations appeared variable across schools. In other words, many of the commonly endorsed accommodations (e.g., reduced workloads, extended deadlines, extended time limits for taking tests, alternatives to traditional testing) could require an IEP or 504 plan in some schools.

In order to explore differences between different school communities (i.e., poverty level, racial and ethnic diversity, and urbanicity) and available services or accommodations, we conducted chi-square analyses (see Supplementary Tables S2 and S3). For school services, significant differences were only found between schools in counties with higher versus lower levels of poverty, with self-monitoring instruction (i.e., a strategy to self-assess behavior) significantly more likely to be available in schools in communities with lower levels of poverty $\left(\chi^{2}=7.12, p=0.008\right)$. For accommodations, significant differences were only found between schools in rural counties compared to schools in urban or suburban counties, with missing work forgiveness $\left(\chi^{2}=\right.$ 7.81, $p=0.005)$ and open-book tests $\left(\chi^{2}=6.64, p=0.010\right)$ significantly more likely to be available in schools in rural counties compared to schools in urban or suburban counties. In sum, there were very few significant differences in availability of services and accommodations based on school community differences.

\section{Phase 2 Results}

A total of 19 school professionals were interviewed about their experiences and perceptions of school re-entry. Participants worked in counties considered to be low poverty $(n=16)$ or high poverty $(n=3)$; suburban $(n=9)$, urban $(n=3)$, or rural $(n=7)$; and worked in schools serving predominantly white students and families $(n=14)$ or predominantly ethnic and racial minoritized students and families $(n=5)$. Results from interviews are presented in two sections according to qualitative themes: (1) themes related to school processes for reintegration; and (2) themes related to perceptions of student and family experiences during school reintegration (see Tables 6 and 7 for an overview of each theme and category with illustrative quotes).

\section{Re-Entry Processes}

Themes related to school processes are shown in Table 6, and include four main categories: (1) processes, including whether or not schools have a protocol, re-entry meetings, the development of re-entry plans, and key individuals involved; (2) services, including accommodations and interventions; (3) communication, including contact between the school and members of the student's family, their clinician, and the hospital, as well as between members of the school; and (4) psychosocial considerations.

\section{Re-Entry Protocols, Meetings, and Planning}

Of those $(n=8)$ identifying that their school or school district had a protocol for school re-entry, most $(n=7)$ described it as being followed inconsistently due to internal or 


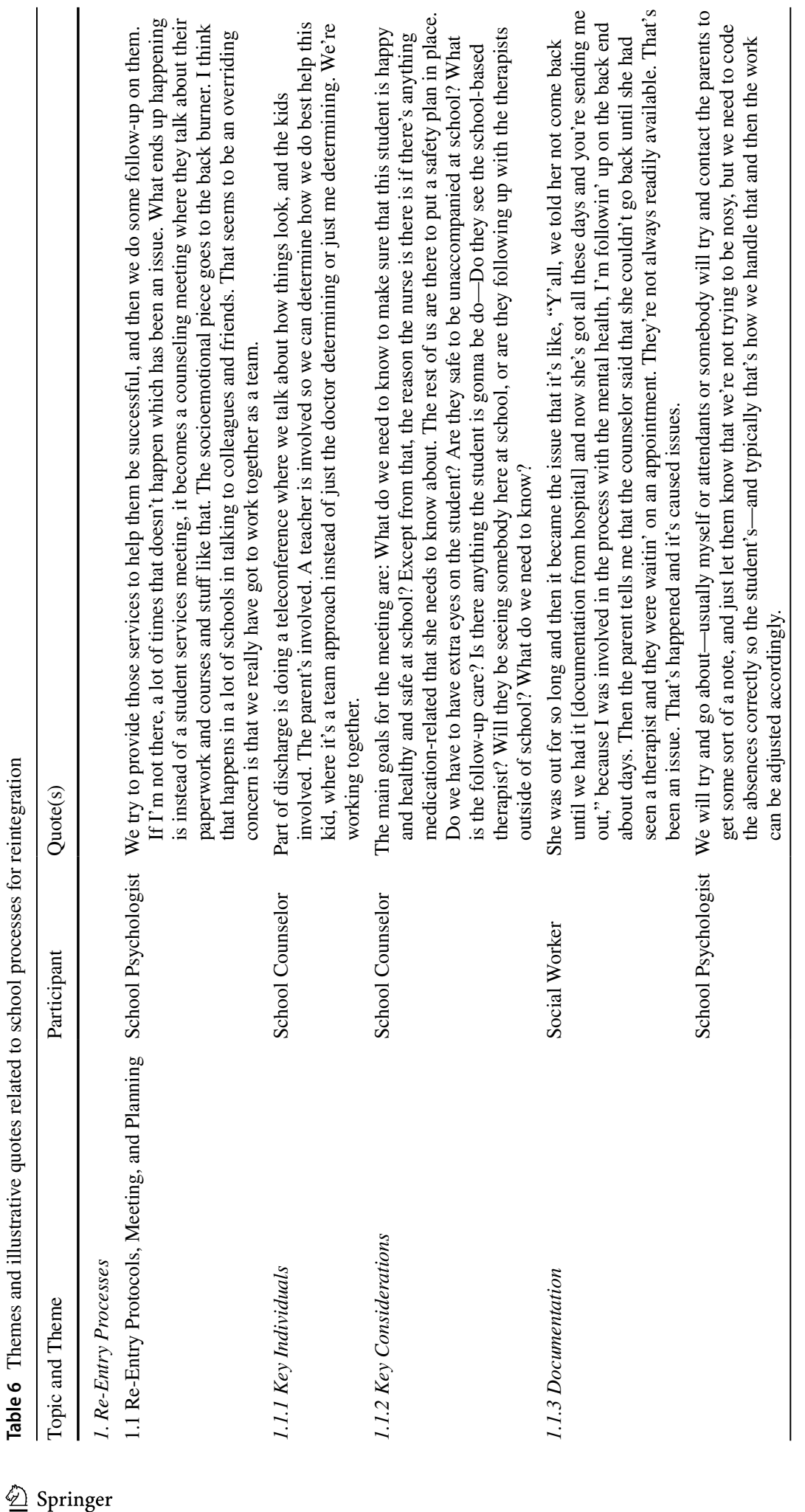




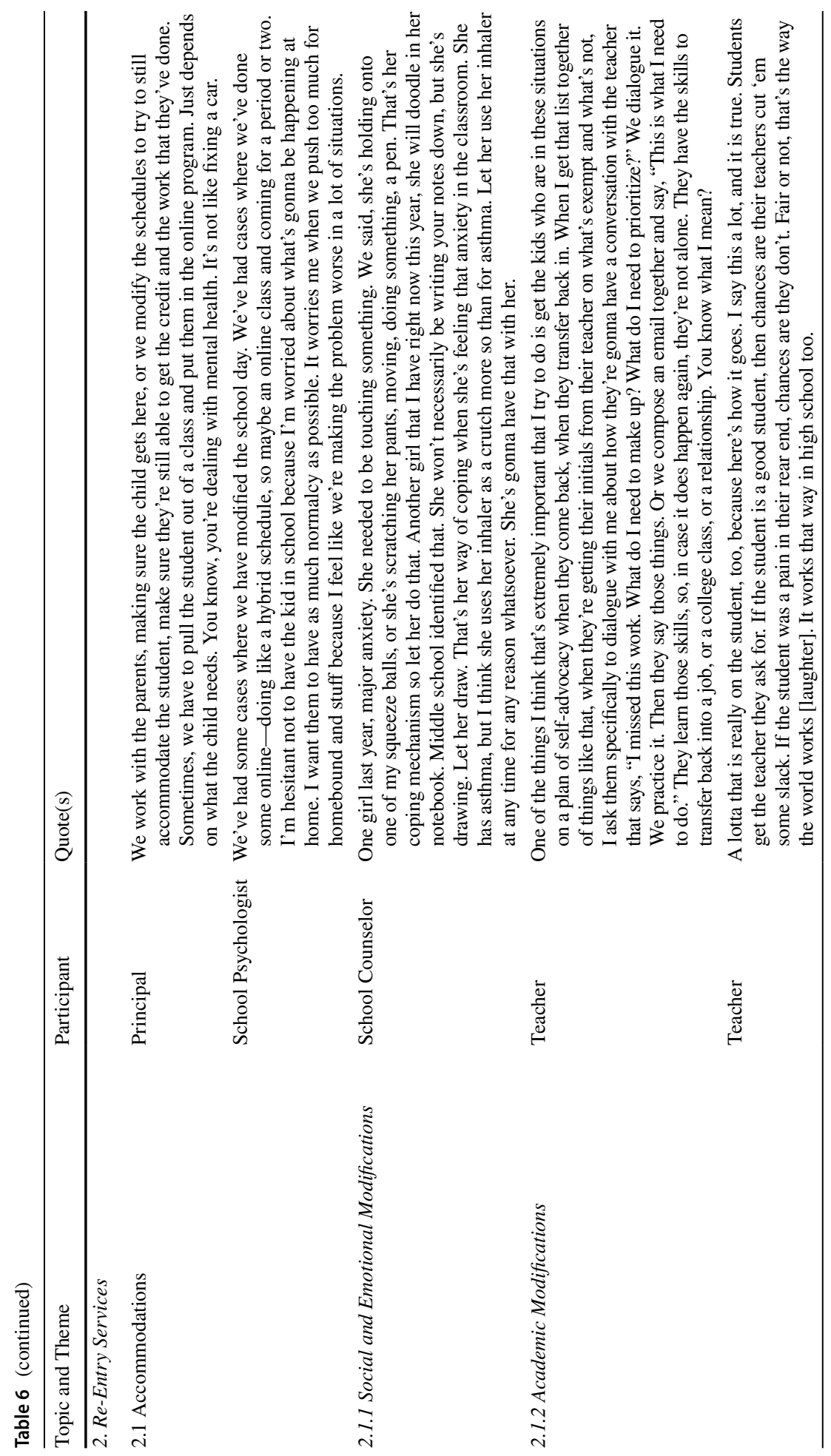




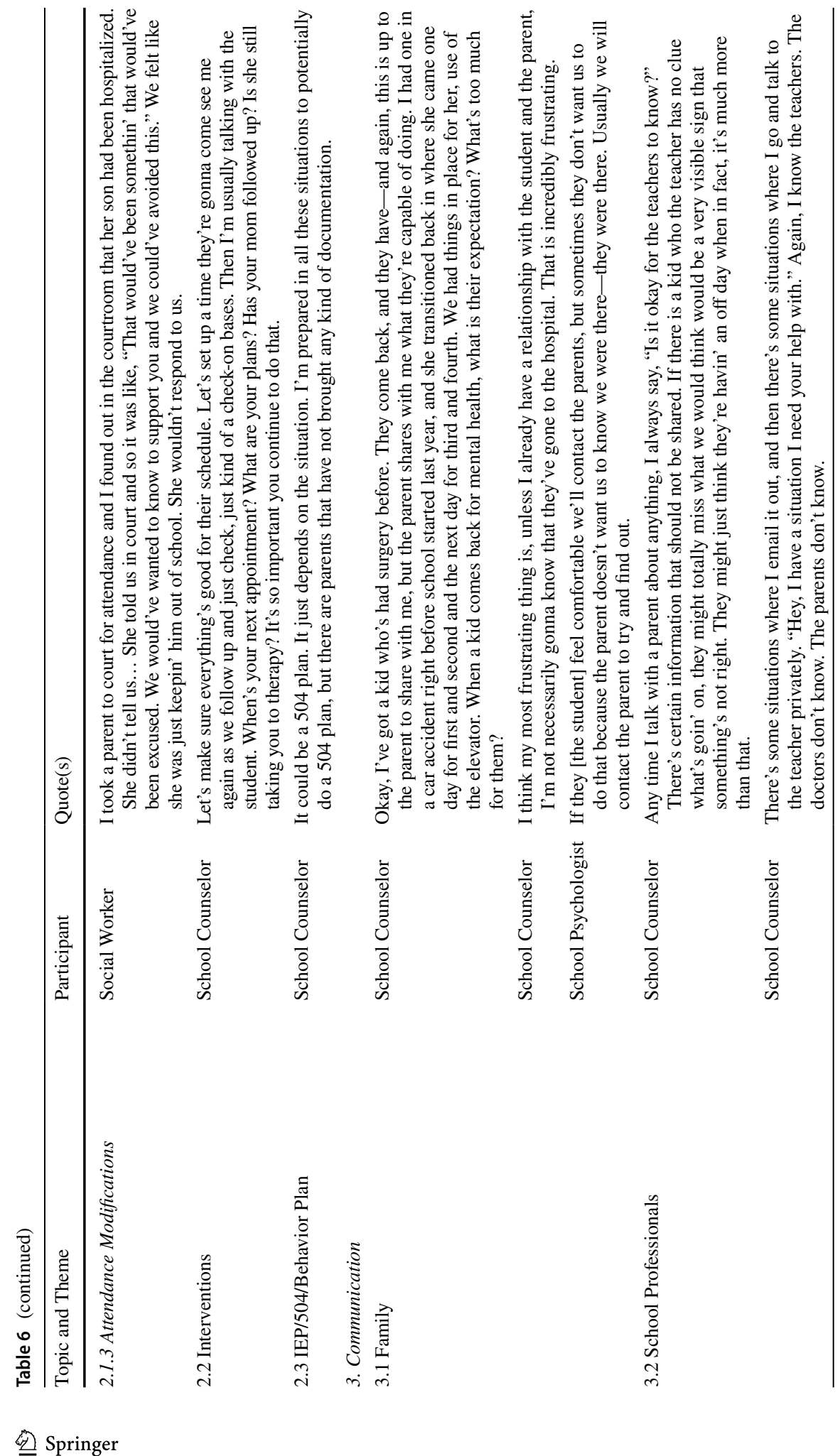




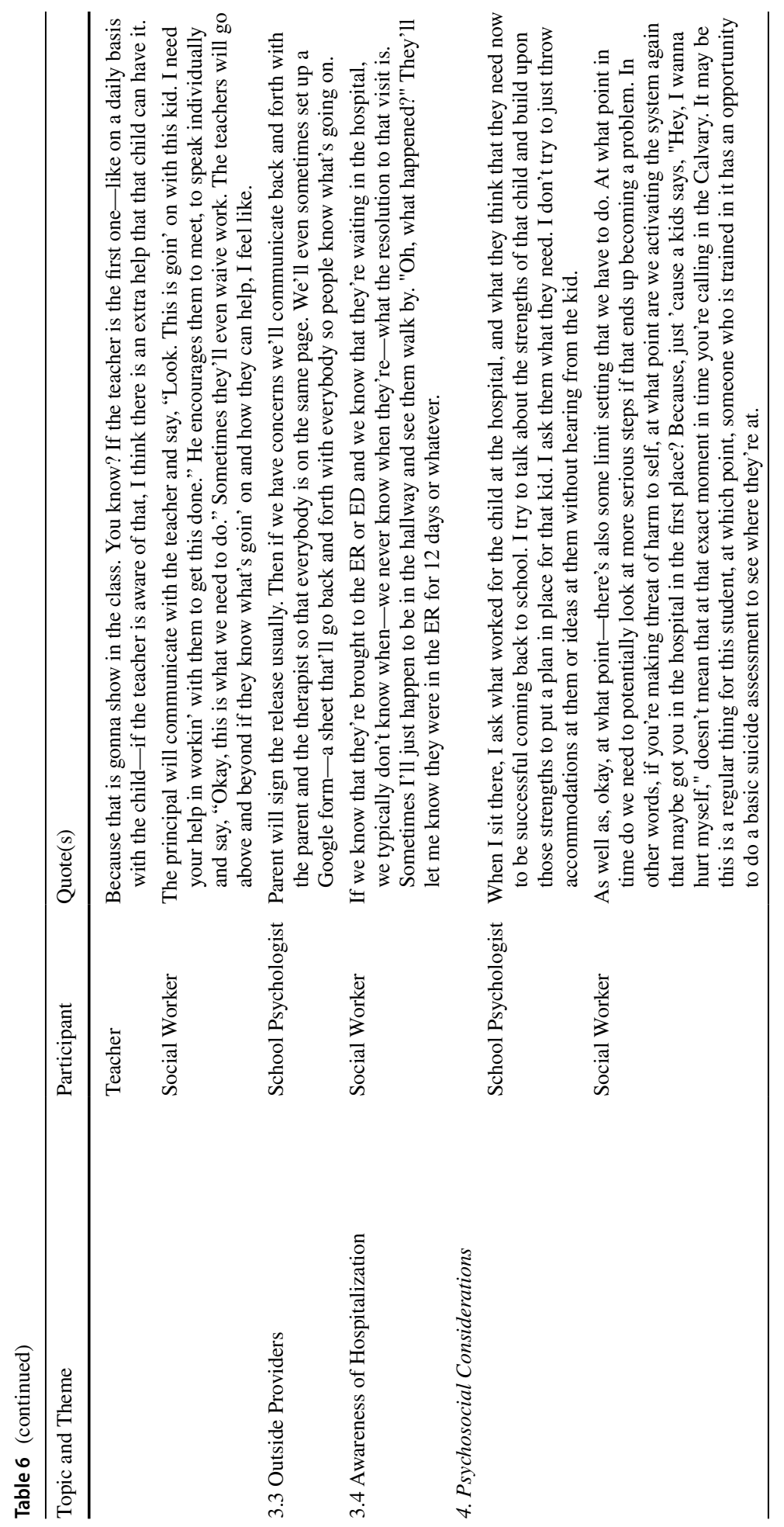




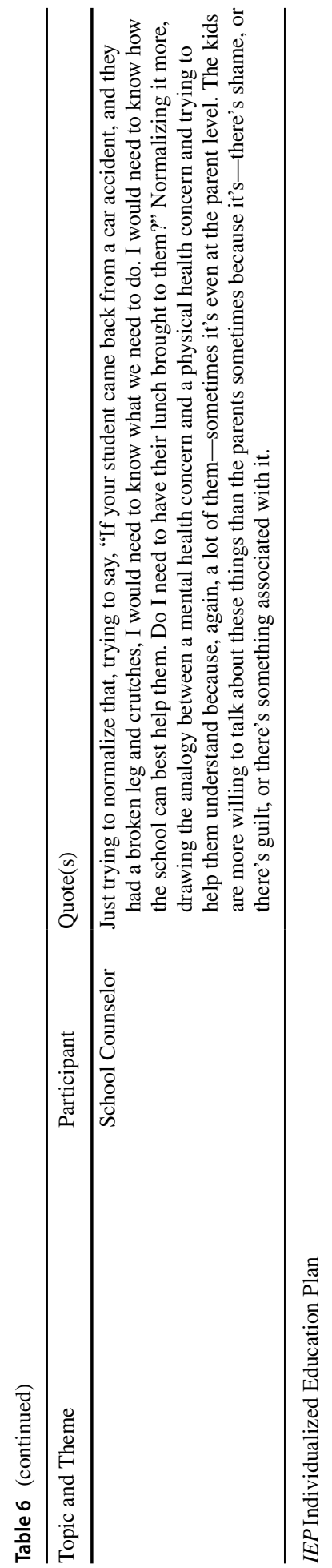




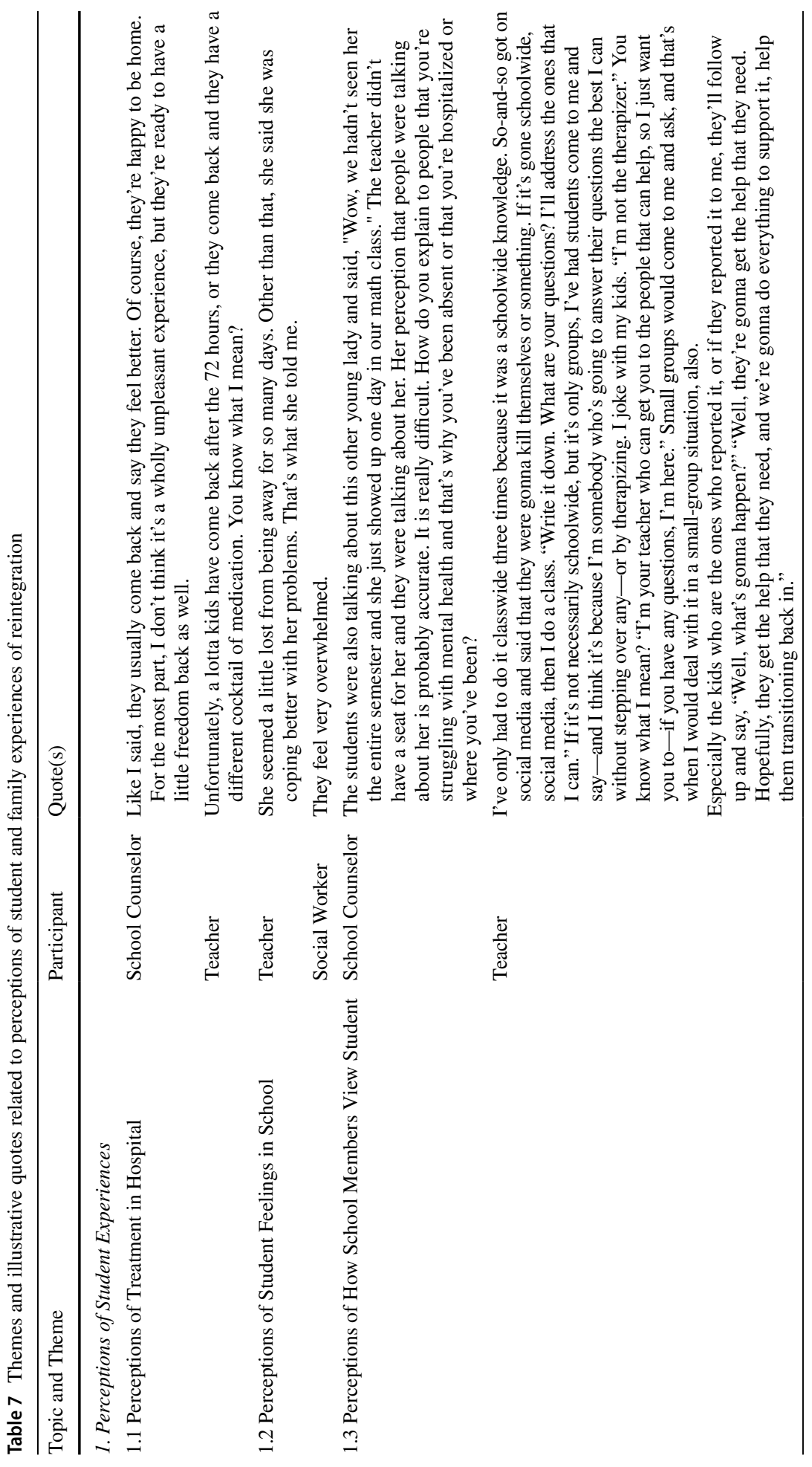




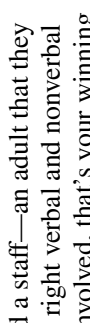

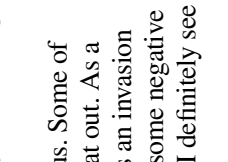

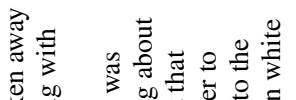

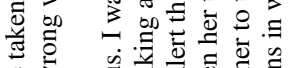

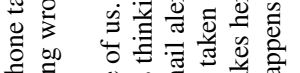

等 0

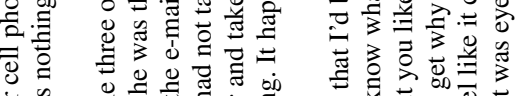

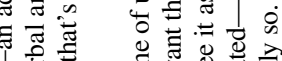

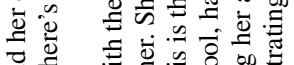

疍

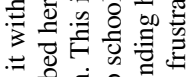

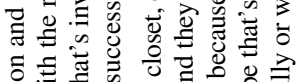

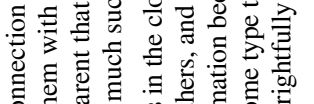

苛范

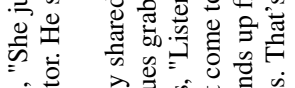

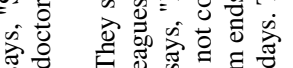

o.

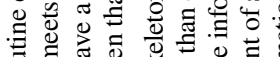

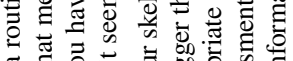

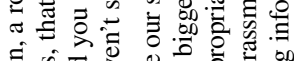

o

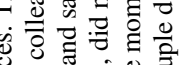

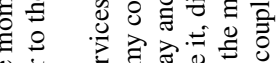

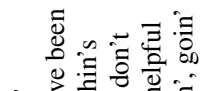

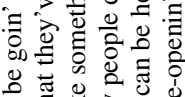

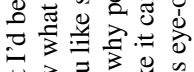

可苛焉

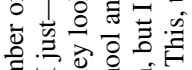

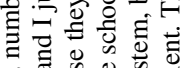

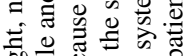

on

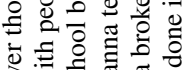

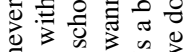

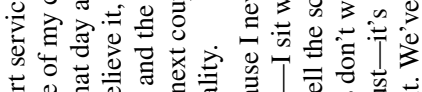

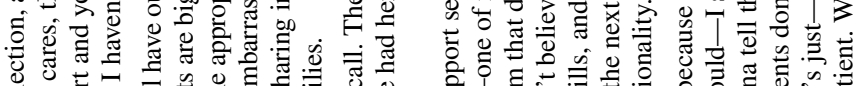

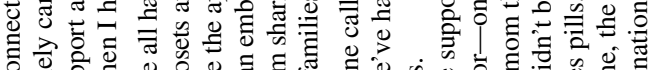

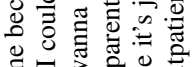

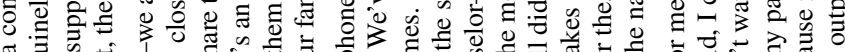

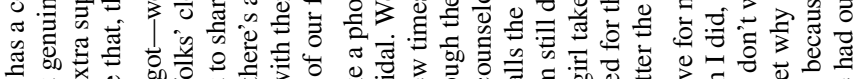

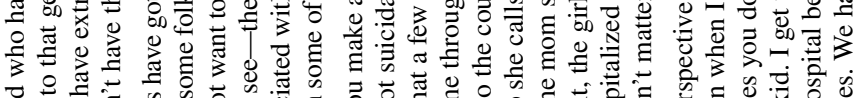

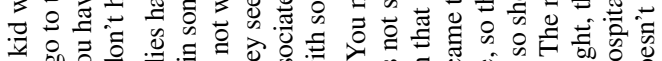

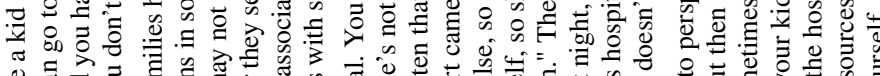

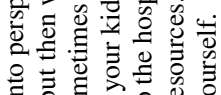

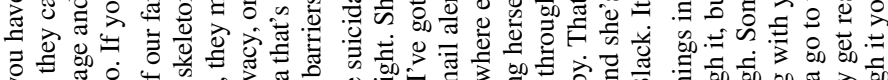

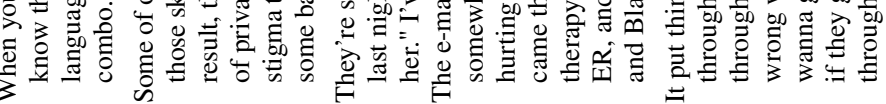

$\overrightarrow{0}$
$\frac{3}{0}$
$\frac{\pi}{0}$
0
0

$\overrightarrow{0}$
$\frac{1}{0}$
$\frac{\pi}{0}$
i

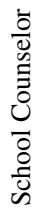




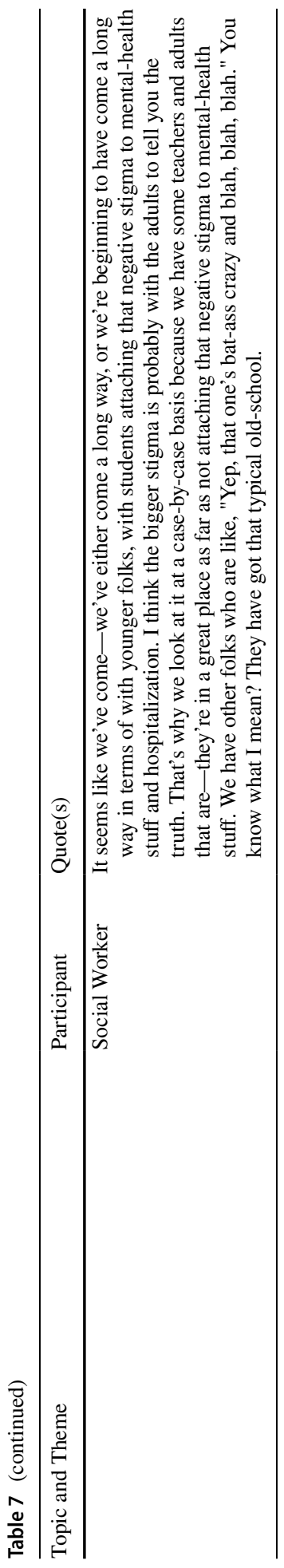


external factors. In particular, three professionals explained that the process hinged on knowing whether or not the student was hospitalized, which is oftentimes unclear. Still others $(n=3)$ described variability according to who was in charge of leading the reentry process, with the focus of the meeting sometimes shifting from social-emotional considerations to academic planning. Of the eight participants explaining their school did not have a protocol (to their knowledge), a few explained that their school had protocols for other emotional and behavioral health concerns (e.g., for risk assessments, extended periods of absences, behavior problems), but not for re-entry.

Thirteen participants described having a re-entry meeting when students return to school from hospitalization, and two explained they were unsure if meetings occurred or believed that they did not occur. Only four explicitly described developing a formal re-entry plan for returning students, although most described similar processes occurring within re-entry meetings or separate, informal plans to support re-entry. Participants described key individuals involved in the process $(n=11)$, key information and considerations they sought when meeting with the student and their family or planning for their return $(n=12)$, and documentation requests or requirements from the hospital $(n=10)$.

Key individuals included parents and families $(n=5)$, social workers $(n=9)$, counselors $(n=9)$, school psychologists $(n=1)$, administrators $(n=5)$, nurses $(n=4)$, students $(n=4)$, case managers or 504 coordinators $(n=2)$, and other $(n=1)$. Two interviewees described how their school takes a team approach to supporting re-entry. Four indicated there was typically a point person or lead to re-entry meetings or planning, identifying the social worker $(n=2)$ or school counselor $(n=2)$.

Multiple participants $(n=12)$ identified information they sought out from students and families to better understand how to support returning students or supportive strategies they employed to help students plan for their return. These included addressing basic information required for planning (e.g., their intended date of return), information about their hospitalization (e.g., length of stay, diagnosis, what worked well in the hospital), ongoing treatment (e.g., medications, outpatient and follow-up care), current functioning, specific triggers and school-related stressors that might be integrated into a safety plan, and school-related supports that they believe may be helpful. Three interviewees specifically identified how they considered hospital recommendations within re-entry planning (if available) and five described making schedule changes based on individual needs.

Participants also described conversations with students and families addressing information sharing within the school. A handful of interviewees $(n=5)$ explained that they ask students and families what information they are comfortable sharing with certain school professionals. Five individuals described supporting the returning student in planning how they would respond to peer questions about their absences generally, or how to prepare for conversations with individuals who may know about what happened already (e.g., if another student referred them). One participant described trying to create a safe environment to try to help support the student's planning. Another, however, described how this process occurred spontaneously in a way that seemed to threaten the students' wellbeing when a nurse abruptly asked the student what they were going to tell their friends. In response, the student "lost it, and then the mother grabbed her up and they stormed out." Finally, two interviewees explained they identify specific school professionals or teachers who students can check-in with during their return, something that was also brought up (and is described in subsequent sections) when describing ongoing interventions. 
The ways in which documentation from the hospital was considered during re-entry planning were described by ten interviewees. Although most simply described attempting to get documentation from the hospital or how it is sometimes or rarely brought in, one interviewee described how their school required a doctor's release for students to return following identification of suicide-related risk. Another explained how they had seen these requirements cause problems at a previous school, preventing the student from returning in a timely fashion.

Others explained how paperwork was rarely brought in, with one explaining, at best it only included "cookie-cutter" recommendations that were "minimal and generic." Ideally, two interviewees described wanting a plan of care or safety plan. A few $(n=3)$ also explained how they also have parents sign an authorization to release information to the hospital or clinician. Finally, a school psychologist explained the importance of documentation in order for returning students to have their absences excused, explaining "we're not trying to be nosy, but we need to code the absences correctly."

\section{Re-Entry Services: Modifications}

Interviewees $(n=12)$ identified a range of social, emotional and academic modifications available to students returning from hospitalization. One interviewee explained that students were relatively good at using the modifications, and another reported they felt the modifications were effective. Regarding the timing of modifications, one interviewee described how after three weeks of support, they check in with students about their needs and progress, adjusting or removing supports as appropriate.

Nine interviewees described accommodations to support returning students that were unrelated to academics. The most commonly identified modification was an abbreviated or modified day $(n=8)$ that could include integration of hybrid or online courses. However, one school psychologist described their concern about allowing students to stay home for too long, preventing them from returning to "normalcy."

Another six participants explained that they offer students universal passes to leave class or provide a safe and quiet place for students to go when needing a break. Other modifications included leaving class early to avoid crowded hallways $(n=1)$, teaching students to self-regulate needed breaks $(n=1)$, changing student seating $(n=1)$ and allowing students to have access to music or sensory objects (e.g., stress ball, drawing; $n=1$ ).

Fifteen interviewees described accommodations specifically addressing academics upon return. Waiving certain aspects of work, providing exemptions for missed work, and reducing homework or overall workloads, were among the most commonly described modifications $(n=13)$. Interviewees $(n=5)$ also described allowing students extended time to submit work. Exemptions and extensions were described as occurring on a case-by-case basis by some $(n=4)$, and as requested of teachers by administrators, other school professionals, and even the hospital $(n=6)$. One teacher described making a work plan with kids, explaining how she further teaches them to self-advocate when communicating with other teachers about their remediation.

Others $(n=4)$ identified challenges around exemptions, including timing (e.g., if students return at the end of the semester, it can be more challenging to navigate exemptions and extensions compared to the beginning), stigma (if students don't share their hospitalization they may not be eligible for extensions or exemptions), and the rigor of the course (advanced placement and honors classes can be less flexible for such accommodations). One counselor explained how even if the school waives work requirements, it can still be a 
challenge to find a way to help the student learn missed material and prepare for required exams, questioning, "What if it's too much for them to stay after school because they're exhausted after just gettin' through a day? What needs to happen?" In contrast, a special education teacher explained that for shorter hospital stays (of one or two weeks), students are usually expected to make up all work, which "is its own bit of stress." This teacher continued to explain how there is often variability across teacher's responses to work remediation and it is up to the students to negotiate with teachers, who often "get the teacher they ask for."

Five interviewees described how they or other school professionals specifically provided workplans with timelines to students and three allowed students to have extended time to make-up work. Other modifications involved allowing students to enroll in a study hall $(n=1)$, providing one-on-one teaching $(n=2)$, and helping students talk to teachers about making up work $(n=2)$. Others $(n=3)$ described how they utilized existing opportunities to support students with making up work (e.g., study halls, tutoring). Specifically, one participant described how all students are eligible to retake tests or receive tutoring from teachers and another described how as part of their school's multi-tiered systems of support (MTSS) process, all teachers hold an open short period where students can connect with them to get caught up or retake missed tests. The third, however, explained that supports like study halls are only available to those with an IEP.

Despite a large focus on academic remediation, a handful of interviewees $(n=4)$ acknowledged the importance of mental health above academics, as well as the ways in which mental health can influence academics. One school psychologist noted the tradeoff between building skills for supporting mental health and the need to stay on top of academics: "If they go into Day Treatment, they stay there long-term, they come back and they may have some coping skills, but now they're behind academically."

Finally, four participants specifically described how their school made exceptions to absences for students returning from the hospital, with three underscoring such exceptions can only be made if the school is informed of the student's hospitalization. For example, a social worker described an incident in which they learned about the student's hospitalization when taking the family to court. The social worker explained how had they known the absences were related to hospitalization, the absences would have been excused and the family would never have ended up in court.

\section{Re-Entry Services: Interventions}

A total of 14 interviewees identified interventions that could be or were provided to returning students. Interventions included the opportunity to participate in school-based mental health $(n=6)$, development and maintaining of a safety plan $(n=3)$, and ongoing monitoring or check-ins with students $(n=10)$. Monitoring and check-ins were described to include check-ins about upcoming appointments and therapy, ongoing counseling sessions, and informal check-ins with teachers or support staff.

A total of 13 interviewees described consideration of IEPs, 504 plans, or behavior intervention plans for returning students. Two explained that unless a student already had an existing IEP or 504, these services would not be considered for returning students (and an additional interviewee explained that while this was true for IEPs, a 504 might be considered). Others $(n=8)$ explained that these services might be considered on a case-by-case basis, with four suggesting that a 504 plan may be particularly useful and "do-able" with a diagnosis. Still, another interviewee cautioned against the utility of a 504 plan for returning 
students, describing it as useful for chronic cases but not acute. Three also described how they would often use the return as a chance to update or revise existing 504 or IEPs. Finally, three participants indicated they might provide a behavior intervention plan for the retuning student, possibly including access to an intensive intervention class.

\section{Communication}

Interviewees identified the importance of communicating during the re-entry process between (a) school professionals and families, (b) other school professionals, and (c) community providers, and hospitals.

Communication between the school and family was described by 13 participants. School Professionals described how parent and caregiver communication was key to information sharing about supporting a student's return. They described contacting families, having families contact them, and the frustration they experienced when families were unwilling to provide any information or notify them of a student's hospitalization. Much of the work interviewees described being able to offer hinged on parent approval and support. For example, one counselor explained how having established relationships from the beginning was critical to communication during reintegration.

Four interviewees spoke to the ways they attempted to communicate with families when students returned to school from the hospital without any warning. Interviewees described making phone calls, conducting home visits, and asking students to communicate with families. Two also described the ways in which they maintained communication with families after a student returns, including follow-up to ensure families have connected with referrals from the hospital.

The ways in which school professionals communicated with other professionals in the building were described by 16 interviewees. Some interviewees described only sharing information with teachers that was identified by students and their families as appropriate to share with; or sharing information filtered down to the essential components to only the essential teachers $(n=8)$. Although two explained that they or their principal meets with the teacher to discuss the student's needs, others $(n=3)$ described teachers learning about hospitalizations through more informal conversations from counselors or administration. As this special education teacher described, these conversations can have implied meanings: "You may get a rumor that 'Hey, just keep an eye on this kid. Make sure he or she's okay,' with air quotes. You know what I mean, but no real specifics, nothin' like that." Still another explained how they, as the counselor, know the teachers and can help determine how and to whom to share information.

Although some described simply sharing with teachers that students were hospitalized, without specifying why, a few explained that teachers usually get a sense of the student's hospitalization because symptoms often show up in class. One professional explained that she felt like very little was communicated to teachers, and another explained that, although it would be helpful for teachers to be made aware of a student's hospitalization, they rarely are. Irrespective of what is shared about the student's mental health, multiple interviewees $(n=5)$ described notifications and communications with teachers about helping students' get caught up, waiving certain work, and being flexible with students.

Eight interviewees described ways in which they communicated with outside clinicians or mental health providers. Most simply explained that they requested waivers to engage in these communications or how they connected with clinicians as part of their process for supporting re-entry, with one noting the barriers to communication presented by federal 
regulations protecting patient and student information (i.e., the Health Insurance Portability and Accountability Act [HIPAA] and the Family Educational Rights and Privacy Act [FERPA]).

Finally, multiple participants $(n=12)$ explained that schools are often not made aware of a student's psychiatric hospitalization. Even if schools made the referral for psychiatric care, interviewees described having limited information about what occurs following their referral. When schools are not told directly (i.e., by parents, as described in family communication), they may find out indirectly or simply guess about a student's circumstance. Eleven interviewees described ways in which they learned about a student's hospitalization when it was not directly from families. They described students re-appearing and telling a school member $(n=6)$, being told about the incident from a peer or "through the grapevine" $(n=3)$, learning about hospitalizations when the hospital school called and requested academic work $(n=2)$, and monitoring student attendance $(n=2)$. Three described accidental ways of learning about students' hospitalizations, with one describing an incident in which their social worker learned about a student's hospitalization because they saw them at the hospital when bringing another student to the hospital.

\section{Psychosocial Considerations}

All interviewees $(n=19)$ described ways they attended to the psychosocial experience of returning youth. A common theme $(n=7)$ was an emphasis on reassuring the returning student, by, for example, sharing their strengths during the re-entry meeting and welcoming them back to school. Five interviewees described the ways in which they tailor supports and services to individual needs and consider diversity of needs when re-entry planning. Considerations included custody arrangements, types of facilities students may be transitioning from, variability in information sharing according to teacher needs, changes in accommodations and interventions based in individuals, and understanding what risk means for each student. Specifically, two interviewees explained how they tried to be sensitive to the different considerations for suicide risk based on individual students, by, for example, questioning "at what point are we activating the system again that maybe got you in the hospital in the first place?".

To help ease students back, some interviewees $(n=4)$ emphasized the importance of making their return natural in order to try to normalize the process and destigmatize their experience. Interviewees also stressed that re-entry supports begin far earlier than when students return from hospitalization, taking more of a prevention approach focused on positive relationships with students $(n=13)$ and families $(n=6)$. As this principal described, building trusting relationships with students and families from the beginning is the foundation from which a positive transition can occur: "every single thing is built off the foundation."

Finally, interviewees also described the importance of teachers showing a sense of caring to students $(n=14)$, and how just like in any setting, some teachers are naturally empathic, and others may be less so. Many described how they believed their teachers (including coaches and teachers involved in extracurricular activities) went above and beyond to support returning students. Still, many also described how much support teachers may need in terms of learning how to be sensitive since they may not be equipped to discuss topics that "make them uncomfortable." A social worker put it simply: "It's easier to talk about homework." 


\section{Perceptions of Students and Families}

Themes related to perceptions of student experiences during school reintegration are shown in Table 7, and include three main categories: (1) perceptions of student and family experiences in school; (2) perceptions of family involvement in supporting the student; and (3) stigma associated with mental health needs.

\section{Perceptions of Student Experiences}

Participants shared their perceptions about the effectiveness of hospital treatment, how they viewed students' and families' experiences during their return to school, and how school members may view the returning students.

Nine interviewees described how they perceived their returning students' psychological functioning in light of inpatient treatment and also how students described their hospital treatment experiences. A handful $(n=5)$ described at least one positive outcome they perceived to be associated with treatment. For example, one interviewee described how students had improved coping skills upon return. Seven interviewees described negative perceptions related to treatment, describing how students appear to receive a "different cocktail of medication," how many have no treatment plan or see very little change, and how some describe feeling like they were treated "like a criminal." Still another was careful to say that long-term care often seemed successful, but short-term or acute care rarely was.

Eight interviewees described how they viewed students' experiences when returning or shared some of the ways in which students described their feelings on return. Most $(n=6)$ explained that returning students seemed primarily embarrassed or worried about what other students might think, or perhaps anxious about what to say to their peers. A special education teacher explained how "the vast majority are embarrassed and afraid somebody's gonna find out where they were or why they were there." Another counselor described how one student felt unwelcome because of increased safety monitoring that meant "people are gonna be watching her and keeping an eye on her."

Four interviewees explained that students often times feel overwhelmed - by the amount of remediation work, the size of their class, or just seeming a "little lost." Likewise, two interviewees acknowledged the stress faced by students and families during reintegration - stress that can be exacerbated by insensitive comments made by school professionals (as described earlier when a nurse asked what the returning student planned to share with peers) and large numbers of school professionals attending meetings.

Interviewees described how teachers and other school adults $(n=5)$, as well as other students $(n=7)$, may perceive returning students. Multiple indicated that for the most part, teachers and staff are understanding, empathic, and nonjudgmental $(n=3)$. Some $(n=3)$ described how stigma may still remain among adults, with a nurse explaining how being so secretive about information sharing contributed to stigma: "It's like hush-hush. It's interesting. We're trying to get away from the stigma of mental health issues. Yet, we as professionals are making it a stigma."

Perceptions of peer reactions were variable. A few explained that kids do notice a student's absence $(n=3)$, whether by rumors or social media. One teacher even described how she led a full class in a writing exercise to help process rumors and questions they had about a hospitalized student. She continued to explain that if peers were the ones to refer the student, it is also important to help answer the questions they have. 
Regarding stigma, one described a high degree among students, another described a low degree, and another school psychologist explained that, among students, it can be "cool to have something wrong sometimes."

\section{Family Involvement}

Multiple interviewees $(n=6)$ expressed the importance of family involvement for a student's recovery following hospitalization. Variability in family involvement was evident from some of the anecdotal experiences interviewees described. A small number $(n=3)$ described specific scenarios in which parents were open, communicative, and supportive, or simply explained they found that caregivers were generally engaged. A handful $(n=5)$ described specific incidents or made general comments about caregivers' lack of involvement. Most of these participants, as well as others, $(n=6)$ described a general variability in caregiver engagement, with a range in degree of involvement from caregivers described as "helicopter parents" to those who may be less "savvy" about their student's needs.

The reasons caregivers may appear less engaged were described by 11 interviewees. They described stigma within the family $(n=6)$ that spanned across different socioeconomic statuses and ethnic/racial backgrounds (e.g., ethnic and racial minoritized families and "helicopter parents"). Three felt some families were in denial or in a state of disbelief about their child, with one describing how the consequences of such denial led to a serious suicide attempt for one student. A couple $(n=2)$ explained how some caregivers were fearful of involvement with the Department of Social Services and two also felt that some families just wanted a return to normalcy or were too busy to be able to address their child's needs. Language barriers were identified as an obstacle to engagement by another four interviewees. And another three explained some families just weren't as "savvy," with one counselor explaining how important it is to support these families with "more hand-holding and walking them through the whole process."

A social worker explained how facing some of these experiences in her own life helped her see how stigma in school served as an obstacle to family engagement, because "they look at you like something's wrong with your kid." Finally, a few described how caregivers could be overly involved $(n=3)$ with one explaining that those of higher socioeconomic statuses were more likely to leverage existing therapists for supporting kids with STB risk.

\section{Stigma}

A final emergent theme included perceptions of stigma for returning students. Across themes, stigma was identified as a concern among families $(n=6)$, students $(n=1)$, and school professionals $(n=3)$. Two also simply described stigma as a problem more broadly, implicating school and societal level stigma as a problem.

\section{Discussion}

The current study aimed to identify and describe strategies employed in schools to support adolescent recovery from a suicide-related crisis that could inform the type of information hospitals consider sharing and recommending to schools during the discharge process. Findings from 
school professional survey respondents and interviewees conducted in one southeastern state of the US revealed themes similar to those reported in previous studies. Stigma related to mental health remains a pressing concern across settings [7, 9], limited communication can be a barrier to effective supports and services for returning youth [1,9], and students and families may face enormous stressors when reacclimating to normal routines [5-7]. Moreover, the types of modifications and interventions available to returning students, as well as the individuals (i.e., school counselors) supporting re-entry in schools, identified in the current study appear comparable to findings from those found nationally in our previous works [2].

Findings also contribute new information not addressed in previous studies. Supports and services provided to students appear comparable in schools across diverse communities: from low poverty to high, urban and suburban to rural, and predominantly white to predominantly ethnic and racial minoritized student bodies, professionals reported similar availability of interventions and modifications to support returning students. Qualitative themes drawn from school professional interviews underscore the ways in which schools conduct re-entry meetings and develop re-entry plans, the types of information they consider, and the services they provide. They also highlight the need for integrating psychosocial considerations and sensitivity to diverse family needs into these practices. Collectively, findings outline the practices and procedures that may be available to returning students, from which hospitals can use to inform recommendations to schools during discharge planning. Based on integration of quantitative and qualitative findings, in the following sections we outline considerations for hospital professionals involved in the discharge process for supporting school re-entry (see Table 8).

Table 8 Steps for preparing for school re-entry following discharge

1 Consider Return to School Throughout Hospitalization. Integrate discussions around school-related stressors and supports with students and families into hospital treatment and discharge planning

2 Discuss Information Sharing with Families. Discuss if families would like to share information with school, considering the following:

a. Attendance Policies: Does the school require documentation of hospitalization to return?

b. IEP/504 Plan: Does the returning student have an IEP or 504 that needs to be updated? Does the returning student have a disorder or concern that merits a request for an IEP or 504 evaluation?

c. Re-Entry Meeting: Because many schools do not automatically hold a re-entry meeting for returning students, does the family know how to request a re-entry meeting to identify a re-entry plan?

d. Informal Supports: Would the student benefit from any informal supports that the school can provide?

e. Comfort: Are there individuals at the school that the patient and family trust to initiate the processes with?

The student's school counselor may be appropriate for families without a previous relationship, but consider other individuals that are already trusted by the family

3 Discharge Summary for Schools. Provide a summary and include recommendations for relevant school-related supports and services that can be shared with the school if families provide permission:

a. Summary. Consider identifying the following information for schools to integrate into their re-entry planning process:

i. School-related stressors and triggers that can inform school supports for returning students

ii. Any diagnoses that could be used for formal supports requiring an IEP or 504 plan can be clearly identified iii. Reasons for hospitalization if applicable to school settings

iv. Medications that may impact student's functioning in school or require medication management in school

v. Coping strategies that can be used by students in school

b. Psychoeducation on Hospitalization and Re-Entry Protocols. Provide an overview of goals related to psychiatric hospitalization, and information about general procedures used by schools to facilitate re-entry

c. Safety Planning. Integrate school environment into safety planning procedures (e.g., identify trusted adults in school in addition to those available at home, identify coping strategies that can be appropriate for classroom settings) and consider sharing with school 
Table 8 (continued)

4 Recommendations for Schools. Provide recommendations for schools supporting returning student tailored to patient's needs

a. Recommendations for Modifications. Consider the following modifications available in most schools for returning students, tailored to each student's needs. Also consider the timing of modifications (are these modifications students may need long-term or modifications that should be reassessed regularly?)

i. Gradual Return to School: Would the returning student benefit from beginning with partial days in school and/or supplemental homebound services or should student to begin school with full school days? Why or why not?

ii. Excused Absences

iii. Pass to Attend School Late or Leave School Early: Would the returning student benefit from beginning school or classes late or leaving early to avoid crowded hallways and busy parking lots?

iv. Consideration of Missing Work Forgiveness: How should academic stressors and academic/work avoidance be considered in light of the returning student's recovery? Consider how the timing of the academic year may influence a school's ability to make changes to work and test requirements

Consider the following modifications that may require an IEP or 504 Plan, tailored to each student's needs:

v. Consideration of Extended Deadlines or Reduced Assignments/Work Load*

vi. Opportunity to Retake Tests*

vii. Opportunity to Take Tests in a Quiet Location*

viii. Extended Time Limits for Tests*

b. Recommendations for Interventions. Consider the following interventions available in most schools for returning students, tailored to each student's needs. Also consider the timing of intervention (are these interventions students may need long-term or modifications that should be reassessed regularly?)

i. Support with Work Completion/Time Management: Would the student benefit from support from the school with a formal work completion plan and strategies for managing time?

ii. Tutoring: Is the student describing or showing difficulties in a specific academic content area or with learning material overall?

iii. Access to a Transition Classroom within School (i.e., a separate space for academic and/or social-emotional support): Would it be helpful for the returning student to have access to a transition space if the school has a separate classroom or location with instructional or social and emotional supports available to returning students?

iv. Check-in/Check-out (i.e., regularly occurring meetings with an identified school member to check in about social, emotional, and academic needs and progress)

v. Individual counseling: Would the returning student benefit from regular counseling sessions with a school counselor?

Consider the following interventions that are only available in some schools, tailored to each student's needs:

vi. Self-Monitoring Instruction**

vii. Group Counseling**

viii. Social Skills Groups**

ix. Peer or Adult Mentoring Programs**

x. School-based Mental Health **

5 Consider Variability Across Schools. Be careful to set appropriate expectations for families since schools may vary in the availability and willingness to provide supports and services to returning students

Although available supports and services may vary by school, * indicates a modification that may commonly require an IEP or 504 plan and $* *$ indicates an intervention that may commonly vary according to school resource availability

IEP Individualized Education Planning

\section{Consider Return to School throughout Hospitalization}

In addition to considering school-related stressors throughout hospital treatment, identification of any diagnostic information that may inform school-related supports could help schools provide more appropriate services during reintegration. Because some interventions and supports may only be available to students with an IEP or 504 plan, hospital providers 
may also consider gathering data relevant to IEP or 504 plan evaluations when administering hospital-based assessments. Providers could play a key role in supporting caregivers in determining if they should request consideration of an IEP or 504 plan, and also provide families with information about what an IEP or 504 plan is. As needed, hospital assessments described in discharge summaries could be used by families to initiate requests for an evaluation, especially considering that schools do not appear to routinely consider providing a 504 plan to returning students.

\section{Discuss Information Sharing with Families}

Unfortunately, limited communication between hospitals and schools has been identified as a significant obstruction to caring for youth returning to school [1,9]. Despite school professionals identifying hospital discharge summaries as important for informing their decision-making process in supporting returning students, they have also noted limited access to this information or recommendations provided by hospitals that may not be realistic in school settings [12]. Yet, many participants in the current study indicated that recommendations from hospitals are an integral component to re-entry planning, despite not always knowing when a student was hospitalized. During the discharge process, hospital providers may consider having a conversation with families about the types of information they would like to share with the school and who the best person for the hospital to communicate with may be, integrating this conversation into other forms of psychoeducation supporting their transition from the hospital [47]. It is important that families understand that without knowing that a student has been hospitalized for a suicide-related concern, schools may be unable to provide services that could support their recovery.

Because stigma associated with mental health crises may serve as a barrier to information sharing, this conversation should be sensitive to the family's concerns and involve a discussion on both the merits of sharing some information with the school (e.g., the potential for excused absences, reduced workloads, and mental health services) and also the drawbacks for others (e.g., concerns about stigma). In some cases, hospital providers may be able to help families identify the most important information to share with schools to ease their concerns about confidentiality.

It is critical that hospitals engage with families in this decision-making process without generalizing across individuals, families, and schools. A wide range of factors can play a role in blocking access to care in schools, including stigma associated with mental health care and problems in families, schools, and communities, systemic racism that favors emotional supports for white students and behavioral discipline for ethnic and racial minoritized students; and much more. Still, majority of youth with psychiatric concerns receive mental health interventions in school settings [39], and findings from the current study indicate that school professionals often feel that they have the returning student's best interest in mind. Thus, a focus on preparing families to self-advocate for their returning student in systems that could vary based on individual families, schools, and school professionals, and supporting school professionals by providing recommendations for supporting the returning student's recovery, could go a long way in facilitating a smoother recovery post-discharge. 


\section{Discharge Summaries for Schools}

Findings from the current study also inform the types of information that may be found in discharge summaries and used to inform supports for returning students by schools. Survey respondents and interviewees described some of the key information that they used to inform school-related supports to include reasons for hospitalization, student triggers and warning signs, medications, coping strategies that can be used by students in school, and recommendations for school-related supports. Depending on family comfort, discharge summaries documenting this information could be shared with schools to help with their decision-making process around continued supports for the returning student and for determining eligibility for an IEP or 504 plan (as applicable).

Interviewees described both positive and negative impressions of the effectiveness of psychiatric hospitalization for adolescents experiencing a suicide-related crisis. Although the effectiveness of psychiatric hospitalization is highly debated [48], discharge summaries including an overview of the purpose of hospitalization (i.e., stabilization and safety) and the expectation that recovery is a longer-term process involving ongoing outpatient care following discharge [49], may help school professionals better understand adolescents' treatment. Such information may also reinforce the importance of school supports during reintegration.

Finally, although previous work did not identify significant differences between schools of varying communities in having a protocol for reintegration across the nation [2], findings from the current study suggest that in this southeastern state, rural schools may be less likely to have such protocols. Thus, another important consideration for hospital providers is the extent to which the school their patients return to is prepared to support their return. Because findings from the current study, and previous work (e.g., [1]), outline some of the strategies employed by schools to support student return, hospital providers may also consider integrating some of these approaches into their recommendations for schools (especially those located in rural areas).

For example, providers could recommend that schools hold a re-entry meeting with the student, their family, and other key members of the school and student's support system in order to develop a re-entry plan. Among the pertinent issues to consider, schools may address any school-related stressors or triggers, identify specific adults for the returning student to go to for help, identify coping strategies to reinforce in school, outline a work remediation plan, consider the student's schedule, and consider how best to balance academics with mental health. Moreover, providing the school with a safety plan that accounts for school context, or encouraging schools to collaborate with returning students to develop a safety plan using some of the information provided by hospitals, could help school professionals handle and monitor ongoing risk and minimize unnecessary referrals to the emergency department [50]. School professionals in the current study expressed an interest in receiving safety plans that could be implemented in schools, because, as described by a school social worker, when a student with a history of suicide-risk expresses suicidal ideation or intentions of self-harm, it "doesn't mean that at that exact moment in time you're calling in the Calvary."

\section{Modification and Intervention Recommendations}

Feasible modifications that hospitals may consider recommending to schools for supporting returning students during recovery could include universal pass to connect with a trusted 
adult, missing work forgiveness, and an abbreviated or modified day in school. On the other hand, hospital recommendations may also encourage schools not to use some of these standard accommodations. For example, it is possible that patients struggling with school or work avoidance may actually benefit from returning to a full schedule and delaying their return to school could do more harm than good. By outlining how the returning student's functioning relates to specific recommendations, hospital providers may help the school provide appropriate supports and services tailored to individual student needs.

Interventions could address support with time management and assignment completion, check-in/check-out interventions, and individual counseling. In some cases, schools may offer school-based mental health services providing access to psychotherapy. Importantly, however, schools can also be encouraged to leverage services already integrated into their universal curriculum. For example, schools following a multitiered system of support (MTSS) may be able to leverage existing intervention periods to help students catch up on academic work. Thus, schools may be able to support returning students by thinking creatively about opportunities that are readily available.

\section{Consideration of Variability in School Settings}

Although findings from this study do indicate commonalities in supports and services available across schools, hospital providers should remain cautious in setting patient expectations about what a school can and cannot provide. For example, although absences may be excused for returning students in many schools, policies vary, and absences can still influence a student's academic success. Even with excused absences and forgiven or reduced workloads, students are often still accountable for exams and tests mandated by the school or state. Another factor affecting availability of modifications is the timing of hospitalization within the academic year and the types of courses students are taking. Students hospitalized early or late in the semester may have more flexibility in changing their schedule or handling missed work, but students hospitalized in the middle of the semester may have less. Likewise, students in general education classes may more easily receive accommodations for work exemptions compared to students in advanced or rigorous courses. Thus, psychoeducation to families that encourages them to seek out understanding of the policies and services available in their own school, for their own circumstances, can help students and families hold appropriate expectations for their return.

Irrespective of school resources, schools should be offered a full range of recommendations to support the student's return given that supporting a smooth transition back to school does not necessarily require additional material resources or costs. Certain details from the hospital may bolster processes that are already in place, such as supports available within MTSS and IEP/504 plan evaluation. Although many of the suggestions do require additional time from school professionals (e.g., check in/check out, individual counseling, re-entry meeting), implementing these supports proactively may reduce overall burden on school staff. Specifically, supports may play a role in helping to prevent student crises, academic remediation or drop out, and conflict with families. Hospital professionals should put forth recommendations tailored to the needs of the returning student, explaining that it is each school's prerogative to implement the supports that are feasible for their setting. Providing this guidance to school professionals may better equip them to support the returning student. As one teacher noted, "If we can make it more comfortable for them, I guarantee you, they'll find the time." 


\section{Limitations}

This mixed-methods study integrated the perspectives of school professionals about reintegration supports and services across one southeast state of the US. Because participants were drawn from only one region, results may not generalize to other parts of the US. Findings are based on self-report measures and interviews and may be impacted by self-report bias. Additionally, multiple participants in the survey represented the same school, potentially inflating prevalence estimates of services available across schools and also reflecting inconsistencies in perceptions of school protocols. Although noted as a limitation, these inconsistencies also underscore the importance of a standardized, written protocol that is clearly communicated across professionals. Finally, because this study aimed to identify practical and feasible school-based supports and services, the sample focused only on school professionals and likely overlooked important considerations as perceived by other stakeholders. Inquiries addressing the perspectives of hospital providers, patients, and parents are needed to substantiate the recommendations outlined here.

\section{Conclusion}

As adolescents recovering from a suicide-related crisis return to schools, they may require varying levels of school-related supports and services. When planning for hospital discharge, in addition to considering outpatient treatment and safety in home environments, hospitals should also consider the adolescent's school-related needs, including how and if the family would like to share information about hospitalization with the school, whether they may require an IEP or 504 plan, and the specific types of school interventions and modifications that may be helpful. Findings from the current study inform recommendations that hospitals can provide, reinforcing the importance of tailoring recommendations to individual patient and family needs and the unique context of their school. As this principal explained, the process "Just depends on what the child needs...you're dealing with mental health. It's not like fixing a car."

Supplementary Information The online version contains supplementary material available at https://doi. org/10.1007/s11126-021-09942-7.

Acknowledgements The researchers would like to acknowledge the professionals who donated their time to this project and the Qualitative Science and Methods Training Program (QSMTP) of the Department of Psychiatry and Human Behavior, Warren Alpert Medical School of Brown University, which provided training in qualitative research methods for this manuscript.

Author Contributions All authors have made substantial contributions to the acquisition, analysis, or interpretation of data.

Funding This project was supported by Grant SRG-0-093-17 awarded to Marisa Marraccini from the American Foundation for Suicide Prevention. The project described was also supported by the National Center for Advancing Translational Sciences (NCATS), National Institutes of Health, through Grant Award Number UL1TR002489. Marisa Marraccini's effort was supported by the National Institute of Mental Health (K23MH122775; Marraccini). The content is solely the responsibility of the authors and does not necessarily represent the official views of the American Foundation for Suicide Prevention or NIH. 
Availability of Data and Material The quantitative dataset analyzed for the current study may be available from the corresponding author on reasonable request.

Code Availability All statistical procedures were conducted using standard codes available within SPSS.

\section{Declarations}

Ethics Approval This study was approved by the Institutional Review Board.

Consent to Participate Study participants completed informed consent procedures prior to data collection.

Conflict of Interest The authors have no conflicts of interest or competing interests to disclose.

\section{References}

1. Tougas AM, Rassy J, Frenette-Bergeron É, Marcil K. Lost in transition: a systematic mixed studies review of problems and needs associated with school reintegration after psychiatric hospitalization. Sch Ment Heal. 2019;11(4):629-49.

2. Marraccini ME, Lee S, Chin A. School reintegration post psychiatric hospitalization: Protocols and procedures across the nation. School Ment Health. 2019;11:615-628. https://doi.org/10.1007/ s12310-019-09310-8.

3. Clemens EV, Welfare LE, Williams AM. Tough transitions: Mental health care professionals' perception of the psychiatric hospital to school transition. Resid Treat Child Youth. 2010;27(4):243-63.

4. Clemens EV, Welfare LE, Williams AM. Elements of successful school reentry after psychiatric hospitalization. Prev Sch Fail. 2011;55(4):202-13.

5. Preyde M, Parekh S, Heintzman J. Youths' experiences of school re-integration following psychiatric hospitalization. J Can Acad Child Adolesc Psychiatry. 2018;27(1):22.

6. Preyde M, Parekh S, Warne A, Heintzman J. School reintegration and perceived needs: The perspectives of child and adolescent patients during psychiatric hospitalization. Child Adolesc Social Work J. 2017;34(6):517-26.

7. Marraccini ME, Pittleman C. Returning to School Following Hospitalization for Suicide-Related Thoughts and Behaviors: Recognizing Student Voices for Improving Practice. School Psych Rev. 2021. https://doi.org/10.1080/2372966X.2020.1862628.

8. Blizzard AM, Weiss CL, Wideman R, Stephan SH. Caregiver perspectives during the post inpatient hospital transition: A mixed methods approach. In Child Youth Care Forum. 2016;45:(5):759-80. Springer US.

9. Savina E, Simon J, Lester M. School reintegration following psychiatric hospitalization: An ecological perspective. In Child Youth Care Forum. 2014;43(6):729-46. Springer US. Simon \& Savina.

10. White H, LaFleur J, Houle K, Hyry-Dermith P, Blake SM. Evaluation of a school-based transition program designed to facilitate school reentry following a mental health crisis or psychiatric hospitalization. Psychol Sch. 2017;54(8):868-82.

11. Henry L. Service Providers' Perspectives on Coping Strategies of Discharged Adolescent Psychiatric Patients (Doctoral dissertation, Walden University).

12. Tisdale JM. Psychiatric hospitalization to school transitions: Examining professional perceptions of effectiveness and fidelity. University of Rhode Island. 2014.

13. Clotfelter C, Ladd HF, Vigdor J, Wheeler J. High-poverty schools and the distribution of teachers and principals. NCL Rev. 2006;85:1345.

14. Lacour M, Tissington LD. The effects of poverty on academic achievement. Educ Res Rev. 2011;6(7):522-7.

15. West A. Poverty and educational achievement: why do children from low-income families tend to do less well at school?. Benefits. 2007;15(3):283-97.

16. Burkett CA. Obstructed use: reconceptualizing the mental health (help-seeking) experiences of Black Americans. J Black Psychol. 2017;43(8):813-35. https://doi.org/10.1177/0095798417691381. 
17. Planey AM, Smith SM, Moore S, Walker TD. Barriers and facilitators to mental health help-seeking among African American youth and their families: A systematic review study. Child Youth Serv Rev. 2019;101:190-200.

18. McConnell C. Rural School Counseling: Turning Obstacles into Opportunities. 1994.

19. Lapan RT, Gysbers NC, Stanley B, Pierce ME. Missouri professional school counselors: Ratios matter, especially in high-poverty schools. Prof Sch Couns. 2012;16(2):2156759X0001600207.

20. Lapan RT, Whitcomb SA, Aleman NM. Connecticut professional school counselors: College and career counseling services and smaller ratios benefit students. Prof Sch Couns. 2012;16(2):2156759X0001600206.

21. Monteiro-Leitner J, Asner-Self KK, Milde C, Leitner DW, Skelton D. The role of the rural school counselor: Counselor, counselor-in-training, and principal perceptions. Prof Sch Couns. 2006:248-51.

22. Education Trust, Cratty J, American School Counseling Association. Sch Couns Matter. 2019. American School Counseling Association. https://www.schoolcounselor.org/getmedia/b079d17d6265-4166-a120-3b1f56077649/School-Counselors-Matter.pdf.

23. Fontanella CA, Hiance-Steelesmith DL, Phillips GS, Bridge JA, Lester N, Sweeney HA, Campo JV. Widening rural-urban disparities in youth suicides, United States, 1996-2010. JAMA Pediatr. $2015 ; 169(5): 466-73$.

24. Schorr M, Van Sant W, Jameson JP. Preventing suicide among students in rural schools. In Handbook of Rural School Mental Health. 2017;129-45. Springer, Cham.

25. Hirsch JK, Cukrowicz KC. Suicide in rural areas: an updated review of the literature. J Rural Ment Health. 2014;38(2):65.

26. Angerstein G, Linfield-Spindler S, Payne L. Evaluation of an urban school adolescent suicide program. Sch Psychol Int. 1991;12(1-2):25-48.

27. Zenere FJ III, Lazarus PJ. The decline of youth suicidal behavior in an urban, multicultural public school system following the introduction of a suicide prevention and intervention program. Suicide Life Threat Behav. 1997;27(4):387-403.

28. Alegria M, Vallas M, Pumariega AJ. Racial and ethnic disparities in pediatric mental health. Child Adolesc Psychiatr Clin. 2010;19(4):759-74.

29. Freedenthal S. Racial disparities in mental health service use by adolescents who thought about or attempted suicide. Suicide Life Threat Behav. 2007;37(1):22-34.

30. Nestor BA, Cheek SM, Liu RT. Ethnic and racial differences in mental health service utilization for suicidal ideation and behavior in a nationally representative sample of adolescents. J Affect Disord. 2016;202:197-202.

31. Cummings C, Dyson A, Todd L. Beyond the school gates: Can full service and extended schools overcome disadvantage?. London: Routledge. 2011.

32. Langer DA, Wood JJ, Wood PA, Garland AF, Landsverk J, Hough RL. Mental health service use in schools and non-school-based outpatient settings: Comparing predictors of service use. Sch Ment Health. 2015;7(3):161-73.

33. Lyon AR, Ludwig KA, Vander Stoep A, Gudmundsen G, McCauley E. Patterns and predictors of mental healthcare utilization in schools and other service sectors among adolescents at risk for depression. Sch Ment Health. 2013;5(3):155-65.

34. Aseltine RH, James A, Schilling EA, Glanovsky J. Evaluating the SOS suicide prevention program: a replication and extension. BMC Public Health. 2007;1:1-7.

35. Crepeau-Hobson F. An exploratory study of suicide risk assessment practices in the school setting. Psychol Sch. 2013;50(8):810-22.

36. Kataoka S, Stein BD, Nadeem E, Wong M. Who gets care? Mental health service use following a school-based suicide prevention program. J Am Acad Child Adolesc Psychiatry. 2007;10:1341-8.

37. Guo S, Kim JJ, Bear L, Lau AS. Does depression screening in schools reduce adolescent racial/ethnic disparities in accessing treatment?. J Clin Child Adolesc Psychol. 2017;46(4):523-36.

38. Gamble BE, Lambros KM. Provider perspectives on school-based mental health for urban minority youth: access and services. J Urban Learn Teach Res. 2014;10:25-38.

39. Costello EJ, He JP, Sampson NA, Kessler RC, Merikangas KR. Services for adolescents with psychiatric disorders: 12-month data from the National Comorbidity Survey-Adolescent. Psychiatr Serv. 2014;65(3):359-66.

40. Creswell JW, Klassen AC, Plano Clark VL, Smith KC. Best practices for mixed methods research in the health sciences. Bethesda (Maryland). 2011;2013:541-5.

41. Cornell D, Huang F. Authoritative school climate and high school student risk behavior: a crosssectional multi-level analysis of student self-reports. J Youth Adolesc. 2016;45(11):2246-59.

42. Corp IBM, Released. IBM SPSS Statistics for Macintosh, Version 260. Armonk: IBM Corp. 2019. 
43. Economic Research Service, U.S. Department of Agriculture. Percent of total population in poverty. 2019. https://data.ers.usda.gov/reports.aspx?ID=17826.

44. Mayring P. Qualitative content analysis: Theoretical background and procedures. In Approach Qual Res Math Educ. 2015;365-80. Springer, Dordrecht.

45. QSR International. Pty Ltd., NVivo 12 [software program]. Version 12. 2019.

46. Guest G, MacQueen KM, Namey EE. Applied Thematic Analysis. California. 2012.

47. Loch AA. Discharged from a mental health admission ward: is it safe to go home? A review on the negative outcomes of psychiatric hospitalization. Psychol Res Behav Manag. 2014;7:137.

48. Jobes DA. Clinical assessment and treatment of suicidal risk: a critique of contemporary care and CAMS as a possible remedy. Pract Innov. 2017;2(4):207.

49. Hayes C, Palmer V, Hamilton B, Simons C, Hopwood M. What nonpharmacological therapeutic interventions are provided to adolescents admitted to general mental health inpatient units? A descriptive review. Int J Ment Health Nurses. 2019;28(3):671-86.

50. O’Neill JC, Goldston DB, Kodish T, Yu SH, Lau AS, Asarnow JR. Implementing trauma Informed suicide prevention care in schools: Responding to acute suicide risk. Evid Based Pract Child Adolesc Ment Health. In press. https://doi.org/10.1080/23794925.2021.1917019.

Publisher's Note Springer Nature remains neutral with regard to jurisdictional claims in published maps and institutional affiliations.

Marisa E. Marraccini PhD is an assistant professor in the school psychology program in the School of Education at the University of North Carolina at Chapel Hill and a licensed psychologist. Her research focuses on promoting child and adolescent mental health in school settings with an emphasis on suicide prevention. Her work also aims to integrate consideration of school context into clinical interventions and to improve school supports for adolescents recovering from suicidal crises.

Cari Pittleman MEd is a doctoral student within the school psychology program at the University of North Carolina at Chapel Hill. In her research, Cari explores equitable access to mental health supports in both school and clinical settings. Clinically, Cari has experience working with children and adolescents with developmental disabilities, ADHD, autism, epilepsy, and brain injury. She is a former elementary classroom teacher.

Emily N. Toole MEd, BCBA is a doctoral student within the school psychology program at University of North Carolina at Chapel Hill. Emily's research interests include trauma-informed care, externalizing disorders, and adolescent mental health. In her clinical experiences, Emily has served students with autism spectrum disorders, emotional and behavioral disorders, and youth in foster care through behavioral and mental health supports. Emily received a B.S. from the University of Florida and M.Ed. from Vanderbilt University.

Megan R. Griffard MS, MEd is a PhD. student in Policy, Leadership, and School Improvement in the School of Education at the University of North Carolina at Chapel Hill. Her research primarily focuses on principal leadership during disruptions to schooling, including natural disasters and COVID-19. Megan holds an M.S. from Northwestern University, an M.Ed. from the University of Nevada, Las Vegas, and a B.A. from Boston College. 